\title{
Effects of Heave Washout Filtering on Motion Fidelity and Pilot Control Behavior for a Large Commercial Airliner
}

\author{
A.T. van Wieringen, ${ }^{*}$ D.M. Pool,${ }^{\dagger}$ M.M. van Paassen,${ }^{\ddagger}$ and M. Mulder ${ }^{\S}$ \\ Delft University of Technology, Delft, The Netherlands
}

\begin{abstract}
Due to the significant translational heave motion at the pilot station associated with changes in aircraft pitch attitude, the motion cueing for aircraft pitch maneuvering typically requires significant heave washout filtering. Previous studies that attempted to motivate choices in the motion cueing strategy for pitch maneuvering based on measurements of pilot behavior. For the small conventional aircraft considered in these studies, the results indicated that, despite the fact that pilots were found to adapt their control strategy to changes in heave cueing, the pitch rotation had a dominant influence on pilot behavior during pitch tracking. For large commercial airliners, a relevant application of this research as a lot of commercial pilot training occurs on moving-base simulators, the location of the pilot station is significantly further from the center of aircraft pitch rotation, yielding more pronounced heave motion cues during changes in pitch attitude. This difference, in addition to typically slower pitch dynamics that require more lead equalization, implies the best choice in motion cueing for large aircraft may be significantly different from what would be optimal for smaller aircraft. In this paper, an experiment is described in which pilot behavior is measured in a pitch attitude disturbance-rejection task with a controlled element and motion cueing conditions that are representative for a Boeing $\mathbf{7 4 7}$ aircraft. Different third-order heave washout filter settings were considered, in addition to a variation in the presence of 1-to-1 rotational pitch motion. Significant effects of the applied variation in pitch and heave motion cueing are observed, even though the effect of heave motion feedback is indeed found to be comparatively more important for larger aircraft. Furthermore, a heave motion filter that combined a low gain with low filter phase distortion was found to yield the least effect on pilot behavior, while for heave motion filters with a relatively high gain and high break frequency significantly larger contributions of motion feedback to pilot behavior were observed.
\end{abstract}

\section{Nomenclature}

$\begin{array}{llll}A & \text { Sinusoid amplitude, deg } & H_{a_{z_{i c}}, \delta_{c}} & \text { Aircraft ICR heave dynamics } \\ a_{z} & \text { Heave acceleration, } \mathrm{m} / \mathrm{s}^{2} & H_{a_{z_{s}}, a_{z}} & \text { Simulator heave cueing dynamics } \\ a_{z_{c g}} & \text { C.g. heave acceleration, } \mathrm{m} / \mathrm{s}^{2} & H_{a_{z_{\theta}}, \delta_{c}} & \text { Aircraft pitch heave dynamics } \\ a_{z_{i c r}} & \text { ICR heave acceleration, } \mathrm{m} / \mathrm{s}^{2} & H_{a_{z_{\theta}, i}, \delta_{c}} & \text { Aircraft ICR pitch heave dynamics } \\ a_{z_{s}} & \text { Simulator heave acceleration, } \mathrm{m} / \mathrm{s}^{2} & H_{c} & \text { Controlled element dynamics } \\ a_{z_{\theta}} & \text { Pitch heave acceleration, } \mathrm{m} / \mathrm{s}^{2} & H_{h p} & \text { Heave washout filter dynamics } \\ a_{z_{\theta, i}} & \text { Pitch heave acceleration w.r.t. ICR, } \mathrm{m} / \mathrm{s}^{2} & H_{n m} & \text { Neuromuscular actuation dynamics } \\ e & \text { Tracking error signal, rad } & H_{p_{e}} & \text { Pilot visual response } \\ f_{d} & \text { Disturbance forcing function, rad } & H_{p_{\theta}} & \text { Pilot pitch motion response } \\ f_{t} & \text { Target forcing function, rad } & H_{p_{a_{z}}} & \text { Pilot heave motion response } \\ H(j \omega) & \text { Frequency response function } & H_{\theta, \delta_{c}} & \text { Aircraft pitch dynamics } \\ H(s) & \text { Transfer function } & J & \text { Cost function } \\ H_{a_{z}, \delta_{c}} & \text { Aircraft heave dynamics } & j & \text { Imaginary unit } \\ H_{a_{z_{c g}}, \delta_{c}} & \text { Aircraft c.g. heave dynamics } & K_{h p} & \text { Heave washout filter gain }\end{array}$

\footnotetext{
*MSc. Student, Control and Simulation Division, Faculty of Aerospace Engineering, P.O. Box 5058, 2600GB Delft, The Netherlands; a.t.vanwieringen@student.tudelft.nl.

${ }^{\dagger}$ PhD Student, Control and Simulation Division, Faculty of Aerospace Engineering, P.O. Box 5058, 2600GB Delft, The Netherlands; d.m.pool@tudelft.nl. Member AIAA.

${ }^{\ddagger}$ Associate Professor, Control and Simulation Division, Faculty of Aerospace Engineering, P.O. Box 5058, 2600GB Delft, The Netherlands; m.m.vanpaassen@tudelft.nl. Member AIAA.

$\S$ Professor, Control and Simulation Division, Faculty of Aerospace Engineering, P.O. Box 5058, 2600GB Delft, The Netherlands; m.mulder@tudelft.nl. Member AIAA.
} 


$\begin{array}{llll}K_{p_{m}} & \text { Pilot motion gain } & \theta & \text { Pitch angle, rad } \\ K_{p_{v}} & \text { Pilot visual gain } & \dot{\theta} & \text { Pitch rate, rad/s } \\ K_{\theta} & \text { Optimal control attitude gain } & \ddot{\theta} & \text { Pitch acceleration, rad/s }{ }^{2} \\ K_{\dot{\theta}} & \text { Optimal control rate gain } & \sigma^{2} & \text { Variance } \\ K_{\ddot{\theta}} & \text { Optimal control acceleration gain } & \tau_{m} & \text { Pilot motion time delay, s } \\ l & \text { Distance between c.g. and pilot station, } \mathrm{m} & \tau_{v} & \text { Pilot visual time delay, s } \\ l_{x_{i c r}} & \text { Distance between i.c.r. and c.g., m } & \zeta_{h p} & \text { Heave washout filter damping ratio } \\ l_{x_{p i l}} & \text { Distance between i.c.r. and pilot station, } \mathrm{m} & \zeta_{n m} & \text { Neuromuscular actuation damping ratio } \\ N & \text { Number of sinusoids } & \phi & \text { Sinusoid phase shift, rad } \\ n & \text { Remnant signal, rad } & \varphi_{m} & \text { Open-loop phase margin, deg } \\ Q & \text { Optimal control performance weighing gain } & \omega & \text { Frequency, rad/s } \\ R & \text { Optimal control effort weighing gain } & \omega_{b} & \text { Second washout filter break frequency, rad/s } \\ T_{m} & \text { Measurement interval, s } & \omega_{c} & \text { Open-loop crossover frequency, rad/s } \\ t & \text { Time, s } & \omega_{h p} & \text { Heave washout filter break frequency, rad/s } \\ s & \text { Laplace variable } & \omega_{m} & \text { Measurement base frequency, rad/s } \\ T_{L_{m}} & \text { Pilot motion lead time constant, s } & \omega_{n m} & \text { Neuromuscular actuation frequency, rad/s } \\ T_{L_{v}} & \text { Pilot visual lead time constant, s } & \omega_{s p} & \text { Short period mode natural frequency, rad/s } \\ u & \text { Pilot control signal, rad } & & \\ u_{m} & \text { Motion contribution to control signal, rad } & \text { Subscripts and Superscripts } \\ u_{v} & \text { Visual contribution to control signal, rad } & & \\ \vec{x} & \text { Optimal control problem state vector } & \mathrm{B} 747 & \text { Boeing 747 } \\ S y m b o l s & & \mathrm{CIT} & \text { Cessna Citation I } \\ \delta_{c} & \text { Control column deflection, rad } & d & \text { disturbance forcing function } \\ & & t & \text { target forcing function }\end{array}$

\section{Introduction}

Moving-base flight simulators are heavily used for airline pilot training due to their low cost, flexibility, and the fact that they provide a safe training environment. To ensure the best possible training of low-level flying skills in a simulator, it is important that there is a thorough understanding of important aspects of flight simulation that are relevant for simulating large commercial aircraft. One of the most challenging areas in simulating large commercial aircraft is the motion cueing of the simulator. ${ }^{1-3}$ Motion cues increase the realism of the simulation and some studies report reduced differences in pilot behavior during manual control tasks compared to in-flight behavioral measurements. ${ }^{4}$ Due to the physical limitations of a simulator's motion system, the aircraft motion, and thus the motion cues presented to the pilot, must be filtered using a washout filter and therefore only provide the pilot with an approximation of the true aircraft motion cues.

This study focuses on this issue for pitch attitude control of a large commercial airliner. During conventional aircraft pitch maneuvering pilots are subjected to a combination of (coupled) rotational and translational motion cues. Dominant motion cues are the rotational pitch motion, the heave motion that results directly from the pitch rotation and the fact that the pilots are seated well in front of the center of pitch rotation, and heave motion resulting from variations in the altitude of the aircraft c.g. Using the definitions from Ref. 5, these latter two heave motion components are referred to as "pitch heave" $(\mathrm{PH})$ and "center-of-gravity heave" $(\mathrm{CH})$.

Recent studies performed in the SIMONA Research Simulator (SRS) of the Faculty of Aerospace Engineering at Delft University of Technology (TU Delft) ${ }^{5-7}$ focused on the effects of these different motion cues during pitch attitude control tasks and the optimal strategy for cueing this combined aircraft pitch and heave motion in a flight simulator. These previous experiments considered this problem for a small jet aircraft, a Cessna Citation I, as the results of these studies were to be linked to in-flight measurements taken in TU Delft's laboratory aircraft, a Cessna Citation II. ${ }^{7}$ With respect to training pilots in flight simulators, it is of much interest to also consider this problem for large transport aircraft. However, it is unknown (even unlikely) that the results obtained from investigations that consider motion cueing for a small jet aircraft transfer directly to the case of a large transport aircraft, due to considerable differences in aircraft dynamics and geometry.

This paper therefore considers the problem of the motion cueing for pitch maneuvering, and its effect on pilot behavior and performance, for a typical large transport aircraft. The focus of the current research is on how differences in controlled pitch dynamics and the relative magnitude of the different available motion cues may affect utilization of motion information compared to the earlier Cessna Citation I experiments. Especially the much larger distance 


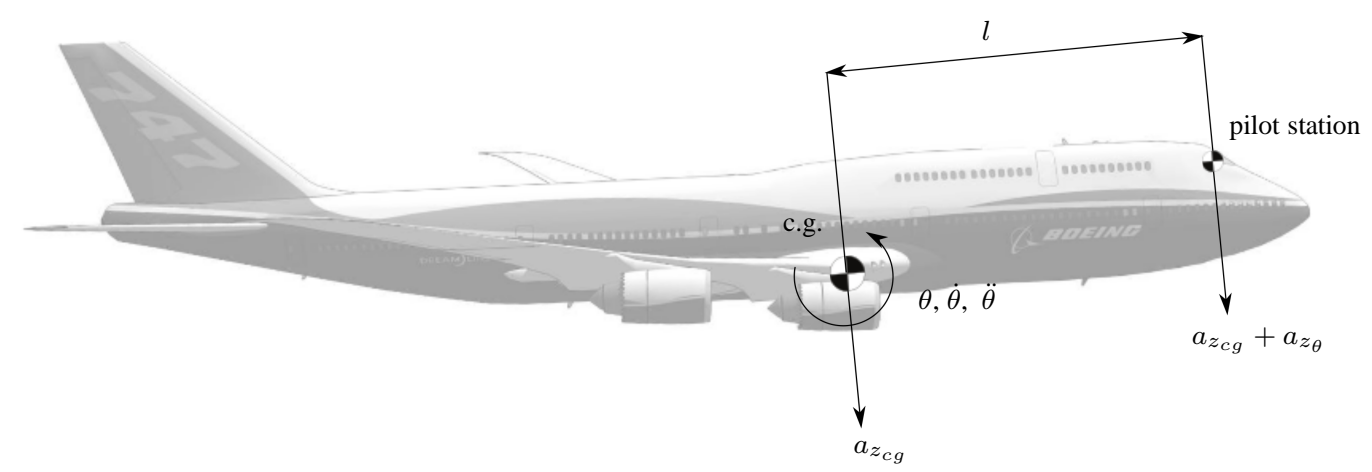

Figure 1. Aircraft motion at the center of gravity and pilot station during a pitch maneuver.

between the center of pitch rotation and the pilot station for large transport aircraft, which results in PH accelerations of much higher magnitude, could be an important factor for deciding on the best cueing strategy for pitch maneuvering. The problem of cueing rotational pitch and translational heave motion is evaluated for a simulator with a typical hexapod motion system, such as the SRS at TU Delft. Due to the fact that a high fidelity mathematical model of a Boeing 747 was available from an earlier study (Ref. 8), this paper will consider this problem for this aircraft. To evaluate extent the results of the experiments of Refs. 5 and 6 can be straightforwardly applied to larger aircraft, a dedicated experiment is performed to obtain measurements of pilot tracking behavior under conditions representative for a large transport aircraft, for a variation in pitch and heave motion cueing settings.

This paper is structured as follows. First Section II provides some background information concerning aircraft pitch control and the motion cues that are perceivable during pitch maneuvering. In addition, an overview of some of the previous studies that used behavioral measurements for evaluating the simulator motion cueing for pitch maneuvers is given. Section III provides an overview of some of the important differences with the small jet aircraft considered in previous studies that need to be considered for large transport aircraft, both in controlled dynamics and in the relative magnitudes of the different motion cues at the pilot station. Section IV describes all the details of the experiment that was performed to evaluate the effect of variations in pitch and heave motion cueing on pilot behavior in the SRS. The results of this experiment are analyzed in Section V and a discussion follows in Section VI. This paper ends with the main conclusions in Section VII.

\section{Background}

\section{II.A. Motion Cues During Pitch Maneuvering}

In conventional fixed-wing aircraft, the pilot station is generally located in front of the aircraft center of gravity. The motion pilots are subjected to during flight are a superposition of movement of the aircraft center of gravity, rigid body rotation around the aircraft center of gravity and possible aeroelastic effects. ${ }^{9}$ This is especially true for aircraft pitch maneuvering, for which a schematic representation of the dominant motion cues that are perceivable at the pilot station is depicted in Fig. 1.

Fig. 1 illustrates that during changes in pitch attitude $\theta$, vertical motion will also be present at the pilot station, in addition to the obvious pitch rotation. For instance, a change in pitch attitude will cause a change in aircraft altitude, yielding relatively low-frequency vertical (heave) motion of the aircraft center of gravity. This component of the symmetrical aircraft motion is referred to as $\mathrm{CH}$ in this study, indicated with the symbol $a_{z_{c g}}$ in Fig. 1. In addition, the pitch rotation around the aircraft c.g. directly induces extra heave motion at the pilot station, due to the position of the pilot station with respect to the aircraft c.g. This latter heave component will be referred to as PH in this study, and denoted with the symbol $a_{z_{\theta}}$. Using the definitions and conventions shown in Fig. 1, and omitting any heave accelerations resulting from aeroelastic modes, the total heave acceleration at the pilot station during pitch maneuvers (in aircraft body axes) can be denoted as:

$$
a_{z}=a_{z_{c g}}+a_{z_{\theta}}=a_{z_{c g}}-l \ddot{\theta}
$$

In Eq. (1), $l$ is the distance in longitudinal direction between the aircraft center of gravity and the pilot station; $\ddot{\theta}$ denotes the pitch acceleration. Note that, despite the fact that there may be an additional small vertical offset between 
aircraft c.g. and pilot station, only the effects of the longitudinal offset $l$ are considered here, as these are typically highly dominant.

Eq. (1) shows that the greater the distance $l$ between pilot station and center of gravity, that is, the larger the aircraft under consideration, the more dominant the pitch heave component $a_{z_{\theta}}$ will be. Some argue that for certain types of aircraft the linear motion cues that result from changes in aircraft attitude even dominate pilots' perception of rotational motion. ${ }^{10}$ As will be shown in more detail in Section III.C, this is one of the key factors that need to be accounted for when interpreting the results of previous experiments performed for small jet aircraft. ${ }^{5-7}$

\section{II.B. Pitch Attitude Control Task and Pilot Behavior}

In this paper, a compensatory pitch tracking task is used to study the effects of the different motion cues that are present during pitch maneuvering on pilot control behavior. As explained in more detail in Ref. 11, compensatory tracking tasks have been used in many investigations into the effects of motion feedback on pilot behavior. . $^{5,12-15}$ A compensatory tracking task is chosen here for evaluating the effects of the different motion cues that occur during pitch maneuvering as such a control task allows for following a cybernetic approach as defined by Mulder. ${ }^{16}$ Such a cybernetic approach revolves around "studying the fundamental properties of pilot-vehicle interaction centered around information, in particular the information used for control". ${ }^{16}$ Previous research has shown that for compensatory tracking tasks, pilots' responses to visual and motion information can be separated using mathematical models of pilot behavior and corresponding identification techniques, ${ }^{13,17}$ thereby allowing for quantitative evaluation and comparison of how pilots make use of these different cues during manual control tasks.

A schematic representation of the pitch control task considered in this paper is depicted in Fig. 2. Fig. 2 shows the pilot controlling the aircraft pitch dynamics in a feedback configuration. Pilot control action is induced using two forcing functions: a target forcing function $f_{t}$, defining the reference pitch that is to be followed, and a disturbance forcing function $f_{d}$, which acts as a disturbance on the controlled element. In this study, these forcing function signals are independent quasi-random sum-of-sine signals, which are known to allow for separating two pilot's responses using frequency domain identification techniques. ${ }^{13}$

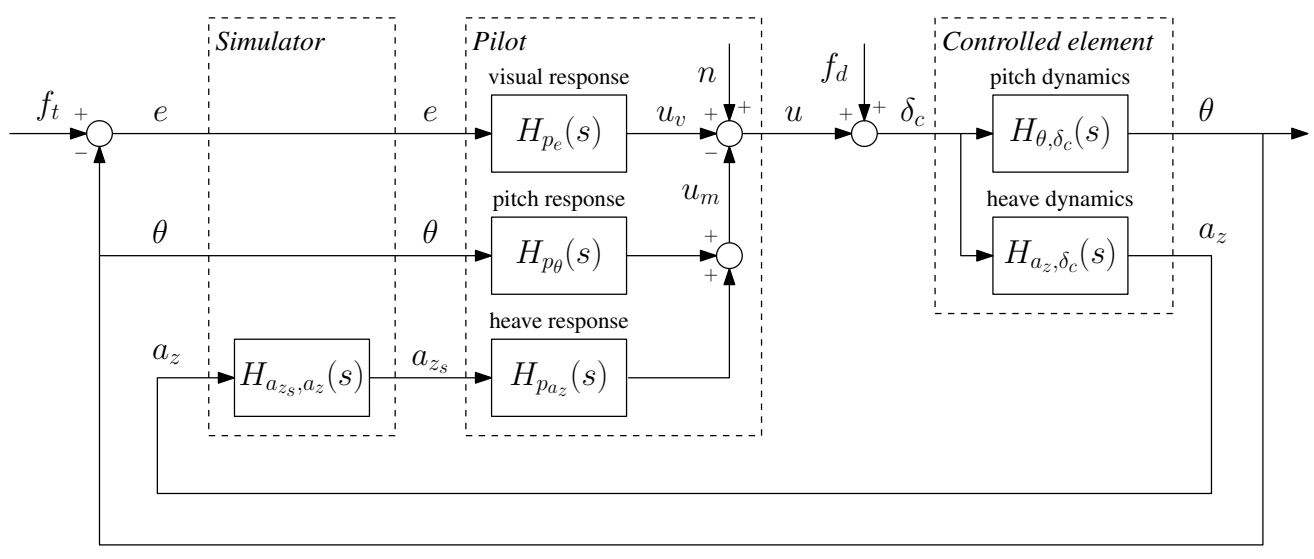

Figure 2. Schematic overview of the closed-loop compensatory control task and model.

Fig. 2 shows that the controlled element for a pitch control task consists of two parts: the column-to-pitch dynamics $H_{\theta, \delta_{c}}(s)$ whose output is the controlled pitch attitude $\theta$ and the column-to-heave-acceleration dynamics $H_{a_{z}, \delta_{c}}(s)$, which define the dynamics of the heave motion that is perceived during the pitch maneuver. Note that $H_{a_{z}, \delta_{c}}(s)$ in this case includes both PH and $\mathrm{CH}$ components (see Eq. (1)). The dynamics of the heave motion filter, which affect the output of the aircraft heave response $H_{a_{z}, \delta_{c}}(s)$ before it is perceived by the pilot, are depicted in Fig. 2 with the symbol $H_{a_{z_{s}}, a_{z}}(s)$. Note that no filtering is considered in this study for the rotational pitch motion cues.

Finally, Fig. 2 shows that the pilot's control behavior is represented as a combination of three separate responses to visually presented tracking errors $H_{p_{e}}(s)$, rotational pitch motion $H_{p_{\theta}}(s)$, and translational heave motion $H_{p_{a_{z}}}(s)$, supplemented with a remnant signal $n$ that accounts for all nonlinearities in the pilot's control behavior. Note that the contributions of the pilot visual $\left(H_{p_{e}}(s)\right)$ and motion responses $\left(H_{p_{\theta}}(s)\right.$ and $\left.H_{p_{a_{z}}}(s)\right)$ to the pilot control signal $u$ are indicated in Fig. 2 with the symbols $u_{v}$ and $u_{m}$. The variances of these two signals will be used in Section $\mathrm{V}$ for evaluating the magnitude of the effect of motion feedback for identified multimodal pilot dynamics. 


\section{II.C. Previous Work}

One of the first studies into the effects of motion cueing for pitch maneuvers on pilot performance and behavior was performed by Van Gool and Mooij. ${ }^{4}$ They performed on-ground and in-air pitch tracking tasks in a Beechcraft Queen Air with a body length of $10.8 \mathrm{~m}$. For their pitch control task, Van Gool and Mooij showed that translational motion during pitch tracking tasks lowered the values of the crossover frequency and degraded the pilot-aircraft performance compared to conditions where no translational motion was present. This effect was found to be highly subject-dependent. Van Gool and Mooij stated that proper rotational acceleration simulation is of higher importance to the pilot than linear acceleration. However, in this study the magnitudes of the linear acceleration cues were relatively low (RMS value of $0.05 \mathrm{~m} / \mathrm{s}^{2}$ ) compared to the pitch acceleration cues (RMS value of $2.1 \mathrm{deg} / \mathrm{s}^{2}$ ).

While not strictly a pure pitch maneuver, Gouverneur et al. ${ }^{18}$ performed a study into the effect of heave cueing settings on pilot performance in a lateral offset approach and landing task, using a simulation model of the Boeing Generic Large Transport aircraft. While motion cueing settings in all other degrees of freedom were kept fixed, heave motion cueing was varied over seven different washout settings (including one with a negative gain, yielding inverted heave motion cues). Despite considering a wide range of heave washout settings, Gouverneur et al did not find a statistically significant effect on pilot landing performance. They did, however, find consistently reduced control activity for higher fidelity heave cueing settings.

Steurs et al. ${ }^{19}$ performed compensatory and pursuit target-following tasks in the TU Delft Cessna Citation laboratory aircraft and in the SRS at TU Delft for different pitch and heave motion cueing settings. In this study, the choice was made to use second order heave motion filters, which required comparatively low gains and high break frequencies to transform the aircraft heave motion to motion profiles that would fit in the SRS workspace. This yielded a set of heave motion filters that would qualify as very low fidelity according to criteria defined by Sinacori ${ }^{20}$ and Schroeder. ${ }^{10}$ A clear effect of increased rotational pitch motion gain on task performance and perceived motion fidelity was observed. However, no consistent differences between the different heave cueing settings were found. Unfortunately, the measurements in the SRS were also only performed for one Citation pilot.

Finally, Refs. 5 and 6 describe two recent experiments that investigated the effects of pitch and heave motion cueing on pilot tracking performance and control behavior for a Cessna Citation I, both performed at TU Delft. Zaal et al. ${ }^{5}$ showed that for this small jet aircraft the effects of the rotational pitch motion were the most significant. However, the third-order heave motion filter used for that experiment was tuned to keep the much more high-amplitude $\mathrm{CH}$ motion within the simulator limits and had a filter gain of 0.6 and a dominant second-order break frequency of $1.25 \mathrm{rad} / \mathrm{s}$. While attenuating the $\mathrm{CH}$ motion to remain just within the simulator workspace, this filter reduced the $\mathrm{PH}$ motion in this experiment to a maximum vertical displacement of $5 \mathrm{~cm}$. This was, in hindsight, believed to be the cause of the limited effect of this PH motion as found by Zaal et al. An additional conclusion drawn from this work was that the $\mathrm{CH}$ motion is not used as feedback by pilots during pitch attitude control, but that this motion component does affect pilot behavior, as the addition of $\mathrm{CH}$ motion showed a modest decrease in task performance and a highly significant increase in visual lead, which is opposite of what is to be expected when additional motion feedback is made available.

Due to the fact that the heave motion filter was in fact not required in the experiment of Zaal et al. ${ }^{5}$ for attenuating the PH motion, a second experiment is described by Pool et al. ${ }^{6}$ in which the effect of the heave washout filter used by Zaal et al. on the measured effect of the PH motion in that study was verified. To achieve this, Pool et al. ${ }^{6}$ considered the same pitch tracking task, but the $\mathrm{CH}$ heave motion was omitted. This experiment showed increased tracking performance when the simulator heave motion fidelity was enhanced by increasing the heave washout filter gain and by removing the filter dynamics. Furthermore, Pool et al. showed that the increase in performance was caused by an increase in the gain of pilots' responses to visual and physical motion stimuli and a decrease in the amount of visual lead by the pilots, allowed for due to the fact that the PH motion provided them with a better source of lead. A marked decrease in performance was already visible when the PH motion gain was scaled with a gain of 0.6 (no further washout), which is generally stated to be a quite acceptable motion filter gain setting. ${ }^{6}$

\section{Effects of Aircraft Size}

Most previous studies into the effects of motion cueing for pitch maneuvers, at least those that used multimodal pilot models for evaluating measured changes in pilot behavior that result from changes in cueing settings, ${ }^{5,6}$ have focused on small jet aircraft. A overall size comparison of the Cessna Citation I considered in Refs. 5 and 6 and the Boeing 747 considered in the current study is shown in Fig. 3.

In this paper, the extent to which the results of the studies performed with a Cessna Citation I model are also applicable to larger aircraft, such as the Boeing 747, is addressed. Two issues are likely to complicate such direct 


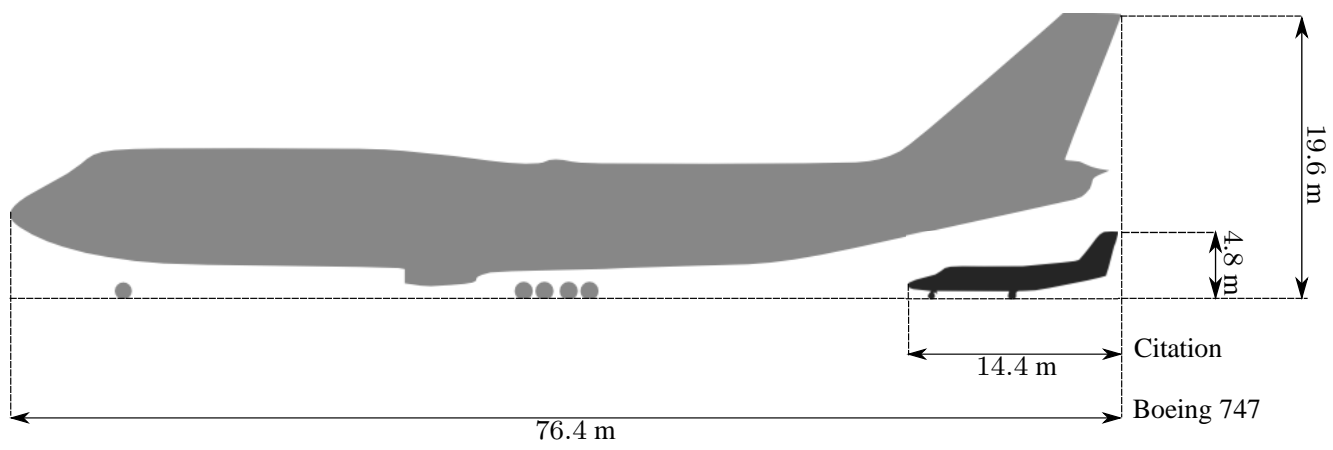

Figure 3. Schematic comparison of Cessna Citation I and Boeing 747 aircraft sizes.

application of these previously obtained results, both resulting from the adaptability of the human pilot to the control task he is to perform. ${ }^{21}$ The first is that larger aircraft typically have slower dynamics than smaller ones, likely resulting in different adaptation of pilot control behavior and, as a result, possibly different effects of motion feedback in general. ${ }^{22}$ The second is that due to the difference in aircraft geometry (most notably a different $l$ ), the relative contributions of $\mathrm{PH}$ and $\mathrm{CH}$ to the total heave motion that is perceivable at the pilot station change. The extent to which these two factors may affect measured pilot behavior, and hence the applicability of the results of previous work to larger aircraft, is discussed in this section.

\section{III.A. Controlled Dynamics}

To evaluate the difference in controlled element dynamics, which will likely lead to a difference in pilot control behavior ${ }^{21}$ during pitch control of a Cessna Citation I and a Boeing 747, here nonlinear models of both aircraft are linearized at the same trim condition to allow for somewhat fair comparison. The chosen trim condition is the flight condition both aircraft would be in at an ILS interception point at 5,000 ft and $160 \mathrm{kts}$. For the Citation I a flap setting of $15 \mathrm{deg}$ was used, whereas for the Boeing 747 flaps were set to $25 \mathrm{deg}$. For the Boeing 747 this resulted in a trim condition with a trim pitch attitude of $3.43 \mathrm{deg}$. The linearized control-column-to-pitch responses $H_{\theta, \delta_{c}}$ (see Fig. 2) of both aircraft, denoted with superscript CIT and B747, respectively, are then obtained as:

$$
\begin{aligned}
H_{\theta, \delta_{c}}^{\mathrm{CIT}}(s) & =\frac{3.11(s+1.13)}{s\left(s^{2}+3.08 s+7.60\right)} \\
H_{\theta, \delta_{c}}^{\mathrm{B} 747}(s) & =\frac{0.77(s+0.67)}{s\left(s^{2}+1.26 s+1.07\right)}
\end{aligned}
$$

Note that the transfer functions given by Eqs. (2) and (3) represent a typical fixed-airspeed (short-period) approximation of conventional aircraft pitch dynamics. Here these equations are defined such that a positive control input $\delta_{c}$ will cause a downward (negative) elevator deflection, which in turn yields a positive pitch-up motion of the aircraft. For comparison of both controlled elements, the frequency responses of Eqs. (2) and (3) are depicted in Fig. 4.

Fig. 4 shows that the Boeing 747 has a lower high-frequency gain, which results in slower response to control inputs. The frequency where both controlled element magnitude responses show a peak, and where they transition from approximately $K / s$ dynamics at low frequencies to $K / s^{2}$ dynamics at higher frequencies, correspond to the natural frequency of the short-period mode. As can be verified from Fig. 4, this short-period mode natural frequency is markedly lower, at $\omega_{s p}=\sqrt{1.07}=1.03 \mathrm{rad} / \mathrm{s}$, for the Boeing 747 than for the Citation I, for which $\omega_{s p}=\sqrt{7.60}=$ $2.76 \mathrm{rad} / \mathrm{s}$. Note that both of these short-period frequencies lie at the lower range of the $2-5 \mathrm{rad} / \mathrm{s}$ expected crossover region for tracking tasks according to McRuer et al. ${ }^{23}$ Due to the lower $\omega_{s p}$, and the requirement for equalizing the controlled pitch dynamics to a single integrator around the crossover frequency, ${ }^{23}$ it can be anticipated that pilots will perform lead equalization starting from lower frequencies - that is, more required lead equalization - for a system with Boeing 747 pitch dynamics than required for the Citation I dynamics given by Eq. (2).

\section{III.B. Optimal Control Analysis}

The comparison of column-to-pitch dynamics frequency responses shown in Fig. 4 suggests pilots would need to perform more lead equalization for the slower Boeing 747 dynamics than would be required for control of the Citation 
(a) Magnitude

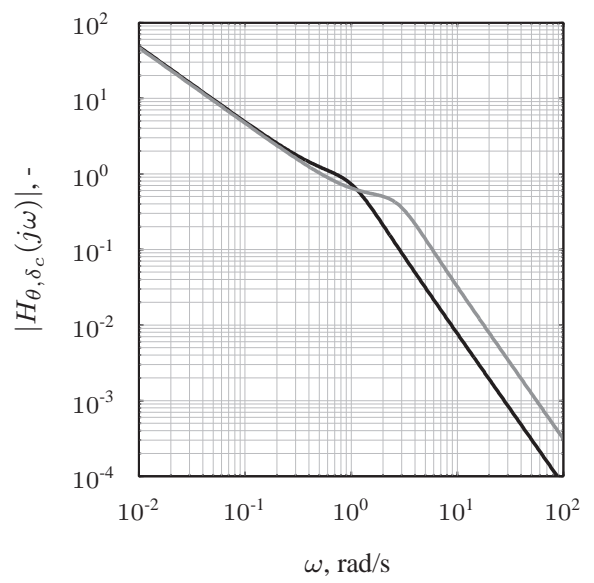

(b) Phase

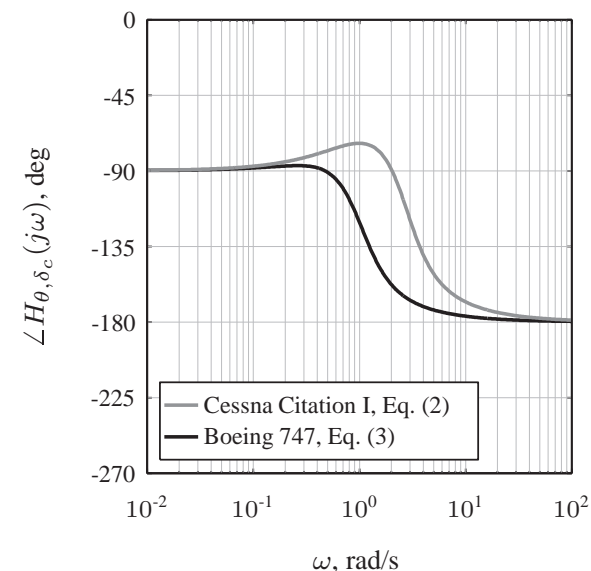

Figure 4. Column-to-pitch dynamics frequency responses for Cessna Citation I and Boeing 747 aircraft.

I dynamics. As effects of motion feedback are typically largest for controlled elements that require more lead equalization, ${ }^{22}$ this could imply motion feedback is more beneficial for the case of Boeing 747 pitch control. Here this observation is quantitatively evaluated by performing an optimal control analysis for the considered pitch control task similar to the one performed in Ref. 5. A schematic representation of the control structure utilized for this analysis is depicted in Fig. 5.

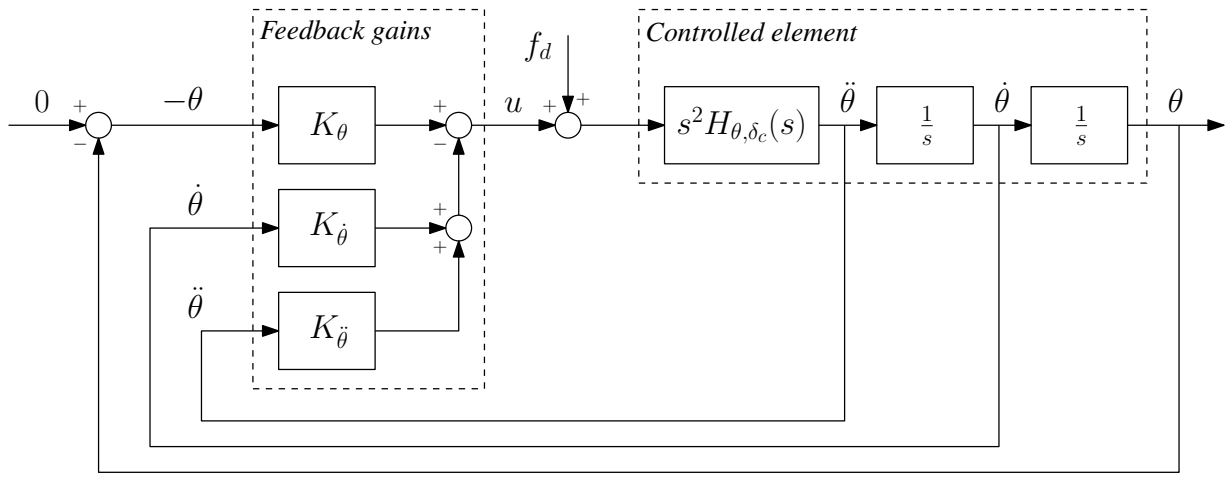

Figure 5. Optimal control analysis pitch control task feedback configuration.

Note that Fig. 5 depicts the same compensatory tracking task as Fig. 2, where the target signal is now set to zero. This implies that pure disturbance-rejection is analyzed here, which is thought to be permissible due to the dominant disturbance signal used in Ref. 5. In addition, the pilot's dynamics have been replaced by the more generic feedback gain structure shown in Fig. 5, which includes separate feedback gains for the pitch attitude, pitch rate, and pitch acceleration. The pitch attitude and its derivatives form the state of the optimal control problem, here defined as $\vec{x}=\left[\begin{array}{lll}\theta & \dot{\theta} & \ddot{\theta}\end{array}\right]^{T}$. From the work of Levison ${ }^{24}$ it is known that a well-trained human controller in optimal conditions can be represented by a controller that tries to find an optimal weighing between performance and effort. In this case a Linear Quadratic Regulator (LQR) was assumed to approximate the pilot where the optimal state feedback gains shown in Fig. 5 are calculated by minimizing the cost function:

$$
J\left(K_{\theta}, K_{\dot{\theta}}, K_{\ddot{\theta}}\right)=\int_{0}^{\infty}\{\underbrace{\vec{x}^{T} Q \vec{x}}_{\text {performance }}+\underbrace{u^{T} R u}_{\text {effort }}\} d t
$$

In Eq. (4) the $Q$ and $R$ matrices are weighting matrices and $u$ is equal to $u=-\left[\begin{array}{lll}K_{\theta} & K_{\dot{\theta}} & K_{\ddot{\theta}}\end{array}\right] \vec{x}$, as can be deduced from Fig. 5. By increasing the value for $R$, the weighing of the input $u$, the resulting feedbacks will result in pilot behavior that lays more emphasis on reduction of effort. To tune the LQR problem solution, the method of Bryson and $\mathrm{Ho}^{25}$ is used, where the values of $Q$ and $R$ are set to the inverse of the squared maximum allowable values of $\vec{x}$ and $u$, respectively. The maximum pitch attitude was set to $\theta_{\max }=5 \mathrm{deg}=0.0873 \mathrm{rad}$, as this is approximately 
the maximum value of $\theta$ that is reached due to the disturbance $f_{d}$. For the maximum pitch rate and pitch acceleration the limits for motion cues generated by the SRS motion base were taken from Ref. $26: \dot{\theta}_{\max }=10 \mathrm{deg} / \mathrm{s}=0.1745$ $\mathrm{rad} / \mathrm{s}$ and $\ddot{\theta}_{\max }=50 \mathrm{deg} / \mathrm{s}^{2}=0.8727 \mathrm{rad} / \mathrm{s}^{2} . Q$ then becomes a $3 \times 3$ diagonal matrix with the square of the maximum allowable state values as the diagonal elements:

$$
Q=\left(\begin{array}{ccc}
\frac{1}{0.0873^{2}} & 0 & 0 \\
0 & \frac{1}{0.1745^{2}} & 0 \\
0 & 0 & \frac{1}{0.8727^{2}}
\end{array}\right)
$$

Similarly $R$, a scalar, is varied from $u_{\min }=1 \mathrm{deg}=0.0175 \mathrm{rad}$ and $u_{\max }=15 \mathrm{deg}=0.2617 \mathrm{rad}$, which are typical values taken from the experiment of Ref. 5. This results in values for $R$ of:

$$
R_{\min }=\frac{1}{0.0175^{2}} \quad \text { and } \quad R_{\max }=\frac{1}{0.2617^{2}}
$$

By solving the Riccati equation for the optimal feedback gains for both the Boeing 747 and Citation I controlled element dynamics $H_{\theta, \delta_{c}}(s)$, values for $K_{\theta}, K_{\dot{\theta}}$ and $K_{\ddot{\theta}}$ are obtained as a function of $R$. These results are depicted in Fig. 6.

(a) Attitude feedback gain

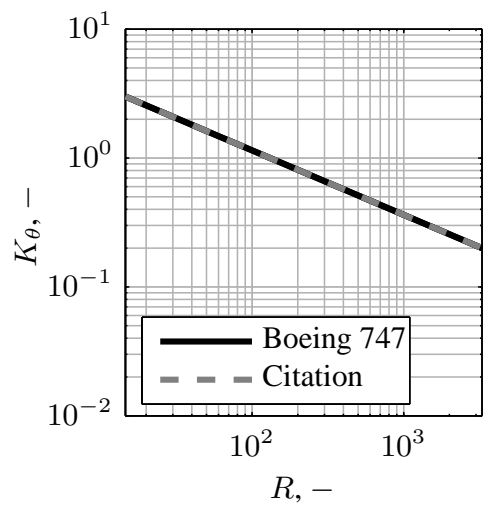

(b) Rate feedback gain

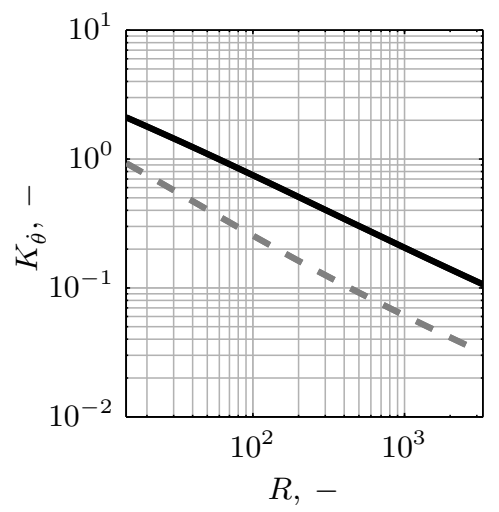

(c) Acceleration feedback gain

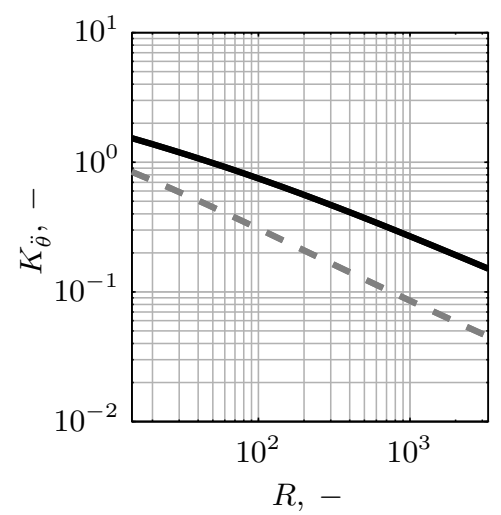

Figure 6. Optimal feedback gains as a function of effort weighing factor $R$ for Cessna Citation and Boeing 747 disturbance-rejection.

First of all note from Fig. 6 that the optimal pitch attitude feedback gain $K_{\theta}$ is of similar magnitude for both aircraft of the whole range of values for $R$. This implies that the feedback of attitude information is of equal importance for control of both aircraft. For the pitch rate feedback gain $K_{\dot{\theta}}$ and the pitch acceleration feedback gain $K_{\ddot{\theta}}$, however, clear differences are observed between the data for the different aircraft dynamics. Both derivative gains are around a factor 2 higher for all values of $R$ for the Boeing 747 controlled element, indicating a stronger reliance on rate and acceleration feedback for optimal control. This confirms the increased requirement for lead equalization for these dynamics due to the lower short-period mode natural frequency $\omega_{s p}$ observed in Fig. 4. As described in more detail in Ref. 5, rotational pitch motion as perceived with the semicircular canals provides pilots with information on $\dot{\theta},{ }^{14}$ which they can use to perform feedback control. In addition, pilots may be able to achieve acceleration feedback from supplied PH motion, as the acceleration perception properties of the otoliths ${ }^{14}$ give them a percept of $a_{z_{\theta}}=-l \ddot{\theta}$. This analysis therefore suggests that the Boeing 747 controlled element might yield more effect of both pitch and heave motion feedback than observed for the Citation I controlled element.

\section{III.C. Heave Response}

Fig. 1 depicts the separation of two different components of the total heave motion that is perceivable at the pilot station as proposed and applied in Refs. 5 and 6. A distinction is made between the heave motion of the aircraft center of gravity $(\mathrm{CH})$ and heave motion of the pilot station with respect to the c.g. (PH). As explained in detail by Field et al., ${ }^{27}$ for a conventional aircraft the pitch rotation that occurs upon a longitudinal column input does not occur around the aircraft center of gravity, but rather around a location commonly referred to as the instantaneous center 
of rotation (ICR), which is typically located closer to the pilot station than the aircraft c.g. By definition, the ICR is the longitudinal along the aircraft longitudinal axis that does not initially move in vertical direction upon a step pitch control input. Especially for large aircraft, the distance between the c.g. and the ICR can be significant. As the transfer function for the $\mathrm{CH}$ heave motion shown in Ref. 5 shows, the aircraft c.g. tends to move down slightly upon a positive column input $\delta_{c}$, yielding non minimum-phase $\mathrm{CH}$ dynamics. The position of the ICR with respect to the longitudinal location of the c.g. is calculated according to:

$$
l_{x_{i c r}}=\lim _{s \rightarrow \infty} \frac{H_{a_{z_{c g}}, \delta_{c}}(s)}{s^{2} H_{\theta, \delta_{c}}(s)}
$$

where $l_{x_{i c r}}$ is defined positive forward of the c.g. As indicated by Eq. (7), the ICR is the location along the aircraft longitudinal axis where there is no direct influence of $\ddot{\theta}$ on the perceived heave acceleration. As the distinction between heave motion that is directly related to $\theta$ and motion that is not directly related to $\theta$ is thought to be important for assessing the effect of motion feedback on pilot behavior during pitch control, a different subdivision between the two heave motion components could also be made, splitting $a_{z}$ in vertical motion of the ICR (ICH motion) and vertical motion with respect to the ICR (IPH motion). This is gives the following relations for the total heave response at the pilot station to a column input:

$$
\begin{aligned}
H_{a_{z}, \delta_{c}}(s) & =\overbrace{H_{a_{z_{c g}}, \delta_{c}}(s)}^{\mathrm{CH}}+\overbrace{H_{a_{z_{\theta}}, \delta_{c}}(s)}^{\mathrm{PH}} \\
& =\underbrace{H_{a_{z_{i c r}, \delta_{c}}(s)}}_{\mathrm{ICH}}+\underbrace{H_{a_{z_{\theta, i}, \delta_{c}}(s)}}_{\mathrm{IPH}}
\end{aligned}
$$

It should be noted from Eq. (8) that both conventions still result in the same total heave motion at the pilot station. Evaluating Eq. (7) for both the Cessna Citation I and Boeing 747 controlled elements results in an $l_{x_{i c r}}$ of $0.92 \mathrm{~m}$ and $5.88 \mathrm{~m}$, respectively. The arm of the IPH accelerations $l_{x_{p i l}}$, as used for calculating $a_{z_{\theta, i}}=-l_{x_{p i l}} \ddot{\theta}$, is then given by:

$$
l_{x_{p i l}}=l-l_{x_{i c r}}
$$

In Eq. (9), $l$ indicates the longitudinal distance between the aircraft c.g. and the pilot station, as depicted in Fig. 1. From Eq. (9), $l_{x_{p i l}}$ can be found to be equal to $2.28 \mathrm{~m}$ and $20.33 \mathrm{~m}$ for the Citation I and the Boeing 747 controlled elements, respectively. Using this information and the linearized and trimmed models of both aircraft, the ICH and IPH heave responses of both aircraft are obtained:

$$
\begin{aligned}
& H_{a_{z_{i c r}, \delta_{c}}^{\mathrm{B} 747}}^{\mathrm{B}}(s)=\frac{-42.63}{\left(s^{2}+1.26 s+1.07\right)} \\
& H_{a_{z_{i c r}, \delta_{c}}^{\mathrm{CIT}}}^{\mathrm{C}}(s)=\frac{-291.09}{\left(s^{2}+3.08 s+7.60\right)} \\
& H_{a_{z_{\theta, i}, \delta_{c}}^{\mathrm{B} 747}}^{\mathrm{B}}(s)=\frac{-15.61 s(s+0.67)}{\left(s^{2}+1.26 s+1.07\right)} \\
& H_{a_{z_{\theta, i}}, \delta_{c}}^{\mathrm{CIT}}(s)=\frac{-7.11 s(s+1.13)}{\left(s^{2}+3.08 s+7.595\right)}
\end{aligned}
$$

The frequency responses of the transfer functions defined by Eqs. (10) to (13) are shown in Fig. 7. These frequency responses show a dominant low-frequency contribution of the ICH motion. Important to note here is that the lower $\omega_{s p}$ for the Boeing 747 yields a reduced magnitude of the ICH motion near the expected crossover region such that the IPH motion, which provides more valuable feedback during pitch control, is more prominent. The frequency responses also clearly show the marked gain difference in the IPH responses for the two aircraft: as a result of the much larger $l_{x_{p i l}}$ the IPH motion is nearly an order of magnitude larger for the Boeing 747.

It should be noted for the experiment considered in this paper always the total pilot station heave motion was cued for conditions where heave motion was present. Despite the fact that in the analysis of pilot behavior (see Section V.C.1) the separation in ICH and IPH heave motion components was considered, the remainder of this paper will still use the acronyms "CH" and "PH" to refer to these motion components for consistency with respect to the discussion of the results of previous experiments. 5,6 
(a) Magnitude

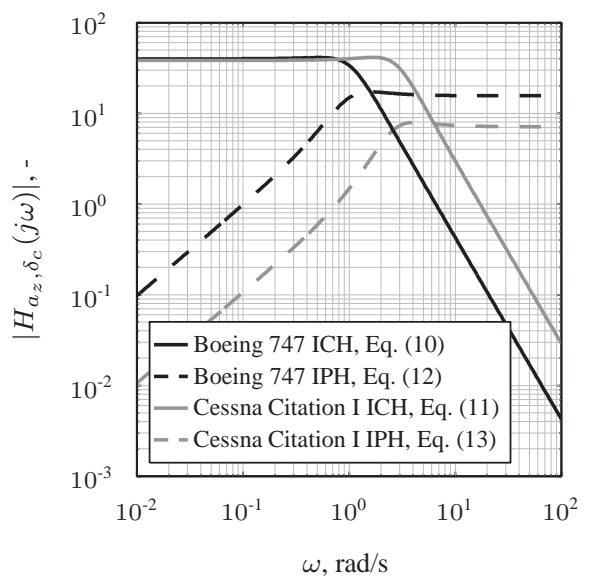

(b) Phase

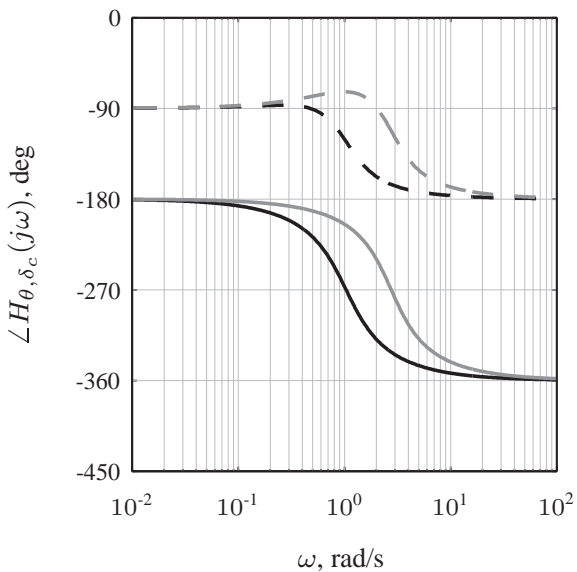

Figure 7. Comparison of ICH and IPH frequency responses of the Cessna Citation I and Boeing 747 models.

\section{Experiment}

To investigate the effects of pitch and heave motion cues as they occur in large transport aircraft on pilot control behavior and performance, a human-in-the-loop experiment was performed in the SRS at TU Delft. The details of this experiment are provided in this section.

\section{IV.A. Apparatus}

The experiment was performed in the SRS at the Faculty of Aerospace Engineering at TU Delft (see Fig. 8a). This simulator has a six-degree-of-freedom hexapod motion system driven by six hydraulic actuators. The time delay of the motion system has been determined to be around $30 \mathrm{~ms} .^{28}$ During the experiment the pilots were seated in the right pilot seat and were operating an active electrical sidestick without breakout force to control the Boeing 747 model dynamics defined by Eq. (3). The sidestick was configured so that only pitch commands could be given. Pitch stick deflections in rad were used as column inputs $\delta_{c}$ to the aircraft model without any further scaling.

(a) Simona Research Simulator.

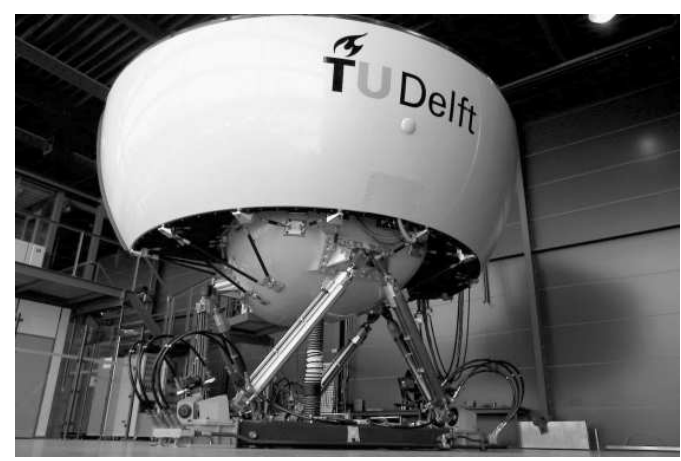

(b) Compensatory display.

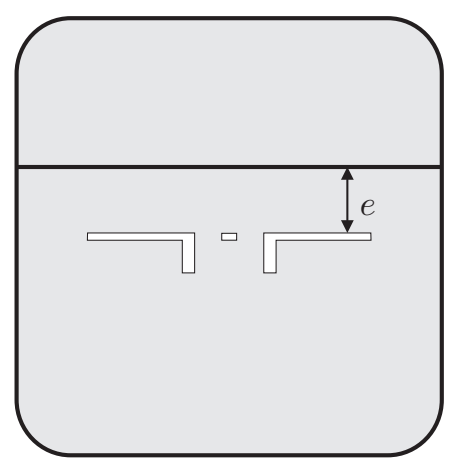

For presenting the instantaneous value of the tracking error in the format depicted in Fig. 8b, use was made of a primary flight display (PFD) located directly in front of the pilot in the SRS cockpit. The update rate of the PFD was $60 \mathrm{~Hz}$. From previous experiments it is known that the latency of the SRS PFD is no more than $25 \mathrm{~ms} .{ }^{29}$ The outside visual system was not used during this experiment and was therefore switched off.

To mask the noise generated by the actuators of the motion system, subjects wore a noise-canceling headset during the experiment. In addition to the noise-cancellation, a masking sound, which consisted of recorded aircraft engine noise, was played over the headphones to conceal any remaining sounds. 


\section{IV.B. Forcing Functions}

In the experiment pilot control behavior was evaluated in the pitch attitude control task defined in Fig. 2. The target and disturbance forcing function signals used in this pitch control task were highly similar to those of the experiments of Refs. 5 and 6. Both signals were defined as quasi-random sum-of-sine signals according to:

$$
f_{d, t}(t)=\sum_{k=1}^{N_{d, t}} A_{d, t}(k) \sin \left(\omega_{d, t}(k) t+\phi_{d, t}(k)\right)
$$

In Eq. (14) $A_{d, t}(k), \omega_{d, t}(k)$ and $\phi_{d, t}(k)$ represent the amplitude, frequency, and phase of the $\mathrm{k}^{\text {th }}$ sine of $f_{d, t}(t)$ respectively. $N_{d, t}$ is the number of individual sine signals in the forcing function signals. Both forcing functions consisted of $N_{d, t}=10$ individual sines. An individual measurement in the experiment was defined to have a measurement time of $T_{m}=81.92 \mathrm{~s}$. To allow for estimating pilot describing functions in the frequency domain, ${ }^{13}$ sinusoid frequencies were all defined to be an integer multiple of the measurement time base frequency, $\omega_{m}=2 \pi / T_{m}=0.0767$ $\mathrm{rad} / \mathrm{s}$.

The forcing function frequencies were chosen slightly differently than those used in the forcing function signals from Refs. 5 and 6, as the sinusoids with the highest frequencies in those signals (around $17 \mathrm{rad} / \mathrm{s}$ ) were found to require extremely high control inputs to follow or attenuate for the Boeing 747 controlled element of Eq. (3). Therefore, these sinusoids were removed from the forcing function signals and replaced with an additional low-frequency sinusoid in both $f_{t}$ and $f_{d}$, below the lowest frequency of the original signals. For the amplitude distribution, the same second order low-pass distribution as used in Refs. 5 and 6 was selected. This amplitude filter is defined as:

$$
A_{d, t}(k)=\left|\frac{\left(1+0.1 j \omega_{d, t}(k)\right)^{2}}{\left(1+0.8 j \omega_{d, t}(k)\right)^{2}}\right|
$$

The phase distribution was chosen by generating a large collection of random phase sets for each forcing function and by selecting the phase set that yields a signal in time that has an approximately Gaussian distribution and no excessive cresting or lack thereof. ${ }^{30}$ As the disturbance signal was added to the control inputs given by the pilot that is, it was inserted before the controlled element dynamics - the disturbance signal amplitudes and phases were preshaped with the inverse of the controlled pitch dynamics, Eq. (3). The amplitudes of $f_{t}$ and $f_{d}$ as calculated from Eq. (15) were scaled to yield time-domain variances for $f_{t}$ and the effective pitch disturbance caused by $f_{d}$ of 0.4 $\operatorname{deg}^{2}$. The full numerical details of the target and disturbance forcing functions are given in Table 1 .

Table 1. Experiment forcing functions.

\begin{tabular}{rrrrrrrr}
\hline \hline \multicolumn{3}{c}{ Disturbance, $f_{d}$} & \multicolumn{5}{c}{ Target, $f_{t}$} \\
\hline$n_{d}$ & $\omega_{d}[\mathrm{rad} / \mathrm{s}]$ & $A_{d}[\mathrm{deg}]$ & $\phi_{d}[\mathrm{rad}]$ & $n_{t}$ & $\omega_{t}[\mathrm{rad} / \mathrm{s}]$ & $A_{t}[\mathrm{deg}]$ & $\phi_{t}[\mathrm{rad}]$ \\
\hline 2 & 0.1534 & 0.3479 & 5.0150 & 3 & 0.2301 & 0.5818 & 3.4793 \\
5 & 0.3835 & 0.7046 & 6.0870 & 6 & 0.4602 & 0.5306 & 1.2876 \\
11 & 0.8437 & 0.9063 & 5.9342 & 13 & 0.9971 & 0.3711 & 6.0892 \\
23 & 1.7641 & 1.4486 & 1.4813 & 27 & 2.0709 & 0.1674 & 5.5074 \\
37 & 2.8379 & 1.9948 & 1.5538 & 41 & 3.1447 & 0.0901 & 1.7336 \\
51 & 3.9117 & 2.3588 & 2.1357 & 53 & 4.0650 & 0.0605 & 2.0192 \\
71 & 5.4456 & 2.8159 & 5.3017 & 73 & 5.5990 & 0.0375 & 0.4411 \\
101 & 7.7466 & 3.5939 & 4.6993 & 103 & 7.9000 & 0.0238 & 5.1749 \\
137 & 10.5078 & 4.7990 & 6.0574 & 139 & 10.6612 & 0.0174 & 3.4146 \\
171 & 13.1155 & 6.2450 & 0.2418 & 194 & 14.8796 & 0.0135 & 1.0656 \\
\hline \hline
\end{tabular}

\section{IV.C. Heave Motion Filter Settings}

In combination with the design of the forcing function signals, the different heave motion filter settings that would be evaluated in the experiment were determined, as the amplitude and frequency distribution of the forcing function signals to a large extent defines the magnitude of the aircraft heave motion that is perceivable during the pitch tracking task of the experiment. As found by Zaal et al. ${ }^{5}$ a third-order high-pass motion filter was required for making sure the mainly low-frequency $\mathrm{CH}$ motion (see Fig. 7) was attenuated enough to allow for performing the tracking task in the SRS without having to resort to extremely low filter gains or very high filter break frequencies. This third-order heave motion filter was of the form: 


$$
H_{h p}(s)=K_{h p} \frac{s^{2}}{s^{2}+2 \zeta_{h p} \omega_{h p} s+\omega_{h p}^{2}} \frac{s}{s+\omega_{b}}
$$

The values for $\omega_{b}$ and $\zeta_{h p}$ were fixed to $0.3 \mathrm{rad} / \mathrm{s}$ and 0.7 , respectively. The filter gain $K_{h p}$ and second-order break frequency $\omega_{h p}$ considered variable over the different heave filter settings that were to be evaluated.

To determine the settings of $K_{h p}$ and $\omega_{h p}$ that would give the highest motion fidelity for the given control task, a set of reference pitch acceleration and heave acceleration trajectories were taken from preliminary human-in-the-loop data collected with subject 1 during the preparation for the experiment. Using this set of reference time traces, an analysis as described by Gouverneur et al. ${ }^{18}$ was performed, where a large number of combinations of $K_{h p}$ and $\omega_{h p}$ were tried to verify if any of the SRS motion base limitations would be reached for a given motion filter setting. The break frequency $\omega_{h p}$ was varied from 0.1 to $4.0 \mathrm{rad} / \mathrm{s}$ with steps of $0.1 \mathrm{rad} / \mathrm{s}$, while values of $K_{h p}$ were tried ranging from 0.1 to 1.0 with steps of 0.05 . This gave a total of 760 different motion filters to be tested. The SRS limits that were considered in this analysis, which were also used in Ref. 18, are given in Table 2.

Table 2. SRS motion system limitations.

\begin{tabular}{lcc}
\hline \hline Actuator state & Limit & Unit \\
\hline Extension & \pm 0.575 & $\mathrm{~m}$ \\
Velocity & 1 & $\mathrm{~m} / \mathrm{s}$ \\
Acceleration & 10 & $\mathrm{~m} / \mathrm{s}^{2}$ \\
\hline \hline
\end{tabular}

Table 3. Selected motion filter settings.

\begin{tabular}{lcc}
\hline \hline Filter & Gain, $K_{h p}$ & Break freq., $\omega_{h p}$ \\
\hline HB & 0.7 & $1.25 \mathrm{rad} / \mathrm{s}$ \\
LG & 0.3 & $0.50 \mathrm{rad} / \mathrm{s}$ \\
HF & 0.6 & $0.85 \mathrm{rad} / \mathrm{s}$ \\
\hline \hline
\end{tabular}

Using the same format as adopted in Ref. 18, Fig. 8 presents the results of this analysis of the 760 possible motion filter settings. Each data point in this graph, which plots the motion filter gain and phase distortion at $1 \mathrm{rad} / \mathrm{s}$ as proposed by Sinacori, ${ }^{20}$ corresponds to a certain combination of $K_{h p}$ and $\omega_{h p}$. The solid black lines in Fig. 8 depict the fidelity boundaries defined by Schroeder $^{10}$ for translational motion cueing. Motion filter settings for which actuator extension limits were reached are indicated with crosses, while filters for which velocity limitations were breached are indicated with square markers. No acceleration limitations were reached for the motion profile considered for this control task. Filter settings that do not interfere with any of the motion base limitations listed in Table 2, and are therefore options to evaluate in this experiment, are depicted with black dots.

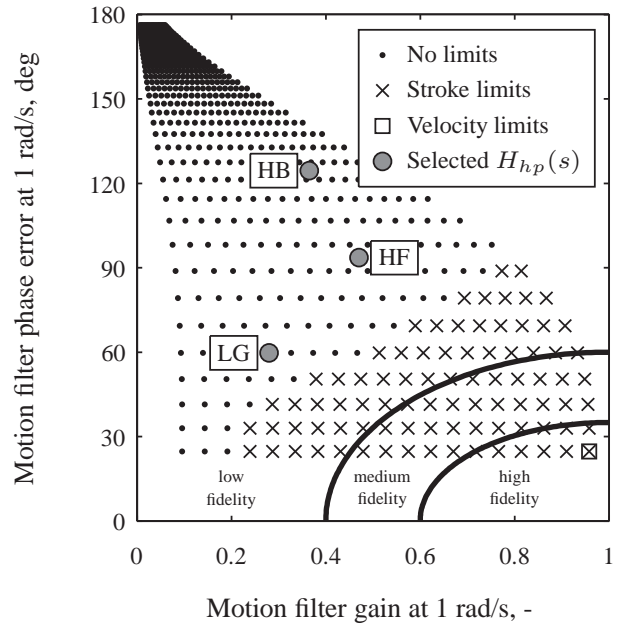

Figure 8. Results of simulator workspace analysis and selected motion filter conditions.

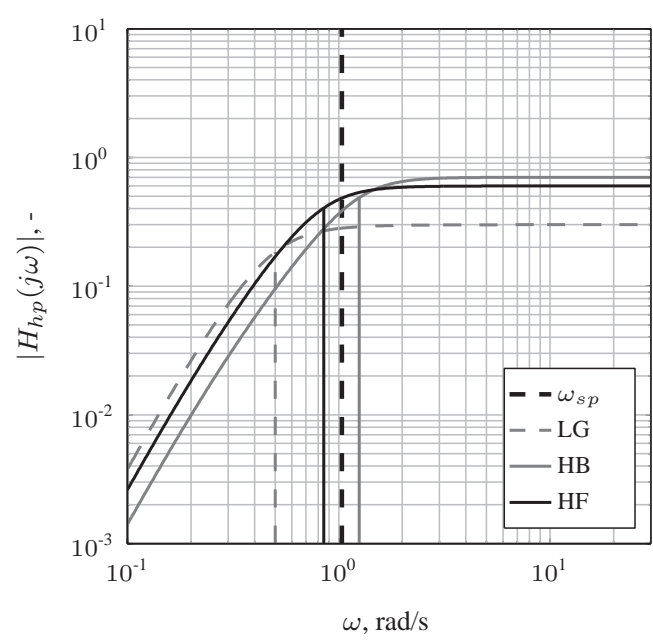

Figure 9. Heave washout filter frequency responses compared to short-period mode natural frequency.

The three final heave motion filter settings that were selected for evaluation in this experiment are depicted in Fig. 8 with gray-filled circular markers. The numerical values of $K_{h p}$ and $\omega_{h p}$ that correspond to these different filter settings are listed in Table 3. The three selected filter settings represent the inevitable trade-off that needs to be made for high-pass motion filters between gain and filter break frequency. The first filter condition has a relatively high filter break frequency of $1.25 \mathrm{rad} / \mathrm{s}$ and is therefore referred to with the acronym HB. As can be verified from Table 3 , this high value for $\omega_{h p}$ also allows for the adoption of a relatively high filter gain of 0.7 . The second filter condition, referred to as LG (for low gain) represents the opposite choice that can be made for the filter parameters. This filter has 
a very low gain, which also allows for limiting the phase distortion of the supplied heave motion cues by allowing for a comparatively low value of the filter break frequency of $0.5 \mathrm{rad} / \mathrm{s}$. The third filter is thought to represent the nearly optimal result of trading-off gain and break frequency and is therefore referred to as the high-fidelity filter condition (HF). As can be verified from Table 3 this filter combines a relatively high gain of 0.6 with a reasonably low value for $\omega_{h p}$ of $0.85 \mathrm{rad} / \mathrm{s}$. Fig. 9 presents the frequency responses of all selected heave motion filters and compares their magnitude response to the short-period mode natural frequency of the Boeing 747 dynamics, $\omega_{s p}=1.03 \mathrm{rad} / \mathrm{s}$. Note from Fig. 9 that the break frequency of the HF filter is above $\omega_{s p}$, which implies significant gain and phase attenuation (not shown) is already present at this frequency for this filter.

\section{IV.D. Independent Variables}

The experiment was designed to investigate the effects of a variation in the cueing of the perceivable aircraft motion on the pilots' pitch attitude control behavior. Both pitch and heave cueing were varied in the experiment based on experience from and for comparison with results of earlier experiments. ${ }^{5,6}$ The three heave motion filter settings listed in Table 3 were supplemented with a condition with no heave cueing (referred to as NH). Furthermore, the pitch control task was performed with and without additional rotational pitch motion for all heave cueing settings, yielding a total of eight experimental conditions. It should be noted that the additional rotational pitch cues were always presented 1-to-1, so no washout was present in the pitch degree of freedom.

\section{IV.E. Subjects and Instructions}

Eight subjects were invited to perform in this experiment. All subjects were affiliated with TU Delft and the Faculty of Aerospace Engineering. All subjects had experience with similar manual control tasks where physical motion feedback was present from earlier experiments. Four subjects had additional experience as aircraft pilots. Two pilots were experienced Cessna Citation pilots. Only one of the subjects was an experienced Boeing 747 pilot. The subjects' ages ranged from 25 to 72 years old.

Prior to the experiment all subjects received a briefing on the experiment and its objectives. The main instruction they received was that it was their task to attempt to minimize the tracking error they perceived on the display.

\section{IV.F. Experimental Procedure}

Each subject started with a set of training runs to familiarize themselves with the dynamics to be controlled and the different heave and pitch motion settings. This training and familiarization period typically consisted of a total of sixteen tracking runs. During the experiment, including the training, the different cueing conditions were presented in random order according to a balanced Latin square design. After each completed set of sixteen tracking runs, corresponding to two completed repetitions of the eight experimental conditions, a forced short break of 15-30 minutes was taken to avoid fatigue of the participants as much as possible.

Tracking performance was monitored by the experimenter during the experiment. The tracking performance was defined as the RMS of the error signal $e$. Also the RMS of the control signal $u$ was calculated after each run. When the subjects' levels of performance and control activity had stabilized, which indicates that participants had adopted a stationary control strategy, and five repetitions of each condition at a stable performance level had been collected the experiment was terminated. Subjects typically performed a total of 8 or 9 runs for each condition, corresponding to 64-72 tracking runs total, to have a consistent set of five measurement runs.

Each experiment run lasted $100 \mathrm{~s}$, of which the final $81.92 \mathrm{~s}$ were used as measurement data. In the first 3 seconds of each tracking run the simulator was tilted to the trim pitch attitude of the aircraft of 3.43 deg pitch up. In addition, the same 3 seconds were used to fade in the disturbance forcing signal. The tilting of the aircraft to the trim angle was done for every condition, including the conditions without rotational pitch cueing and even for the single-loop condition without any pitch or heave cueing.

\section{IV.G. Dependent Measures}

A number of different dependent measures are considered for this experiment for evaluating the effects of the applied variation in motion cueing. Time traces of the pitch attitude $\theta$, pitch rate $\dot{\theta}$, and pitch acceleration $\ddot{\theta}$ were recorded for each run, together with the error signal $e$, control signal $u$ and different heave acceleration components $a_{z_{\theta}}$ and $a_{z_{c g}}$. Using this data various dependent measures were calculated. The variances of the tracking error and the control signal are considered as measures of pilot performance and control activity, respectively. Furthermore, pilot-vehicle system 
target and disturbance open-loop crossover frequencies and phase margins are calculated from estimated frequency domain describing functions ${ }^{12}$ as measures of pilot-vehicle system performance and stability.

Further quantitative measurements of changes in pilot control strategy are obtained by fitting a multimodal pilot model to the measured experiment time histories. The details of this identification procedure and the adopted pilot model will be given in Section V.C.1. The accuracy of the identified pilot models, and how this varies over the different experimental conditions, is evaluated by considering the model variance accounted for (VAF). The VAF expresses the amount of variance in the measured model output signal (in this case the pilot control signal $u$ ) that is explained by the model in a percentage. Finally, the identified pilot models are used to evaluate the relative contributions of the pilot visual and motion responses to the control inputs given by the pilots, as a measure of pilots' reliance on motion feedback. For this evaluation, the fraction of the variances of the signals $u_{v}$ and $u_{m}$ (see Fig. 2) is taken as a metric.

\section{IV.H. Hypotheses}

From the analysis of the available motion cues during pitch maneuvering in Section II and the optimal control analysis in Section III.B it is anticipated that due to the slower aircraft dynamics, pilot will rely more on feedback of pitch rate and also pitch acceleration during the pitch tracking task than found in the earlier experiments of Refs. 5 and 6. Especially the hypothesized increased reliance on pitch acceleration information, which can only be obtained from perceived PH accelerations, suggests heave motion will be an important cue in this experiment.

Due to the significant offset from the center of rotation for the large aircraft considered in this study, the PH heave motion component will likely be the dominant motion cue that is perceivable at the pilot station. As opposed to findings from Refs. 5 and 6 for a smaller aircraft, this implies that the cueing of rotational pitch information might have a much smaller effect on pilot behavior in the current experiment than found in these previous studies.

With respect to the different heave motion cueing settings considered in this experiment, the following hypotheses are made. Based on the above, especially the PH motion is expected to show a large effect on pilot behavior and performance. The low gain filter condition (LG) is therefore anticipated to show only moderate changes in pilot behavior from the $\mathrm{NH}$ condition, as this filter setting attenuates the PH motion the most. The high break frequency filter (HB) will cause a significant phase shift in the supplied heave acceleration cues, especially since $\omega_{h p}>\omega_{s p}$ for this filter (see Section IV.C), but still provides PH motion at the highest frequencies in the measurement range with the highest gain of all three filter settings. The high fidelity (HF) filter, however, is hypothesized to show the most marked effect of the supplied heave motion on pilot behavior, as it is believed to represent the most optimal trade-off between low phase shift and high gain possible for this pitch control task.

\section{Results}

This section presents the results from the eight subjects that participated in the experiment. Data from all dependent measures is presented for each condition as a mean over all subjects and the corresponding $95 \%$ confidence interval of the mean indicated with variance bars. As the experiment had a within-subjects design, all between-subject variability was removed before calculating the confidence intervals. If allowed for by the data, all presented dependent measures were analyzed using a two-way repeated measures analysis of variance (ANOVA) to evaluate the statistical significance of the effects of the applied variation in pitch and heave motion cueing.

\section{V.A. Tracking Performance and Control Activity}

Fig. 10 presents the average measured tracking performance and control activity. These are expressed in the variances of the error signal $e$ and the control signal $u$, respectively. Data for the conditions without rotational pitch cueing are depicted with white filled markers, while data for the corresponding conditions with pitch cueing are presented with black filled markers. Table 4 lists the corresponding repeated measures ANOVA results.

Fig. 10a shows that the tracking error variance was affected by both the variation in heave motion cueing and the presence of rotational pitch motion. The addition of rotational pitch motion is found to result in a reduction in $\sigma_{e}^{2}$ of a consistent $0.02 \mathrm{deg}^{2}$ for all heave cueing settings. As can be verified from Table 4, this is a highly significant effect.

Better tracking performance is also observed with the addition of heave motion in any form, as the tracking error variance is below the values measured for the $\mathrm{NH}$ condition for all other heave cueing settings. Of the three heave cueing settings, the HB filter, which has the highest filter gain and break frequency, is seen to result in the worst performance. Especially when pitch motion is present, the HF filter setting is found to result in the lowest $\sigma_{e}^{2}$ of all

heave cueing settings. The variation in tracking performance with heave cueing setting is also a highly significant effect, as can be verified from Table 4. As the effect of the variation in heave filter settings is highly similar both 
(a) Error variance

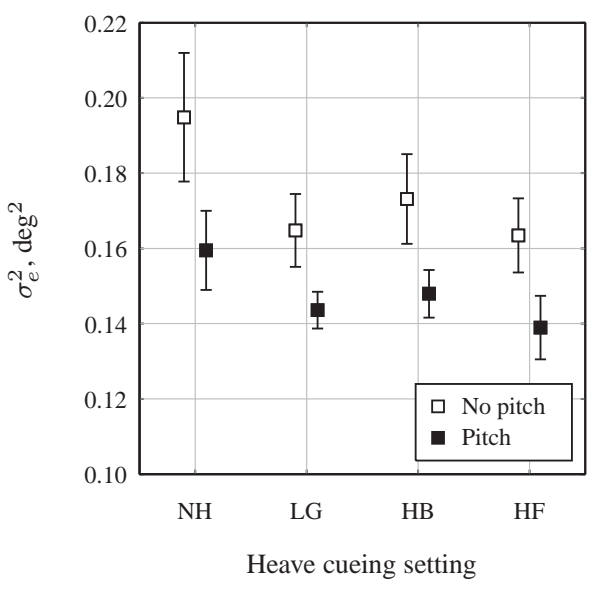

(b) Control variance

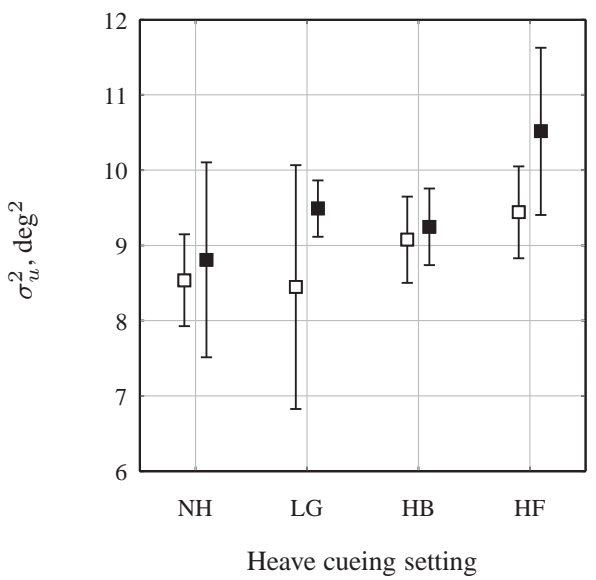

Figure 10. Average error and control signal variances.

Table 4. Within-subject ANOVA results for measured tracking performance.

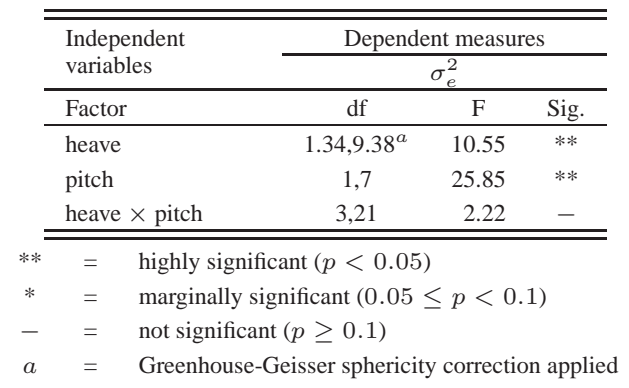

with and without rotational pitch motion, no interaction between both cues appears to be present in the measured performance, which is also confirmed by the ANOVA results.

Measured control activity, which is depicted in Fig. 10b, shows only very slightly increased values for the HB and HF filter settings compared to the NH and LG conditions. In addition, also not too much of an effect of the addition of pitch cueing is visible. Furthermore, due to large differences in the baseline level of control activity between the different participants, a Kolmogorov-Smirnov test on the measured control activity samples showed that the distributions of $\sigma_{u}^{2}$ were significantly different from a Gaussian distribution for all experimental conditions. For this reason, no ANOVA could be performed on the control activity data presented in Fig. 10b.

\section{V.B. Pilot-Vehicle System Open-Loop Characteristics}

For a combined target-following and disturbance-rejection task as considered in this study (see Fig. 2), the pilot has to consider the stability and performance of the combined pilot-vehicle system for both target-following and disturbancerejection. To investigate these properties, the crossover frequencies and phase margins of both the target-following and disturbance-rejection open-loop systems can be considered..$^{12}$ For the mathematical derivation of the equations for these open-loop dynamics, please refer to, for instance, Ref. 5. Fig. 11 shows the average target and disturbance-loop crossover frequencies and phase margins calculated for the current experiment.

Fig. 11a shows the measured disturbance crossover frequencies for all experimental conditions. Typically, better tracking performance (lower $\sigma_{e}^{2}$ ) is associated with a higher crossover frequency. ${ }^{21}$ As can be verified from comparison with Fig. 10a, $\omega_{c, d}$ is indeed found to show nearly perfectly inverse effects of the pitch and heave cueing variation as observed for the tracking error variance. Both the addition of pitch and heave motion is found to yield an increase in disturbance crossover frequency of around $0.3 \mathrm{rad} / \mathrm{s}$. Also in line with the results found for $\sigma_{e}^{2}$, the highest crossover frequencies are found for the LG and HF heave cueing settings. As can be verified from Table 5, which lists the repeated measures ANOVA results for all variables presented in Fig. 11, both the effects of pitch and heave cueing on $\omega_{c, d}$ are found to be highly significant. 
(a) Disturbance crossover frequency

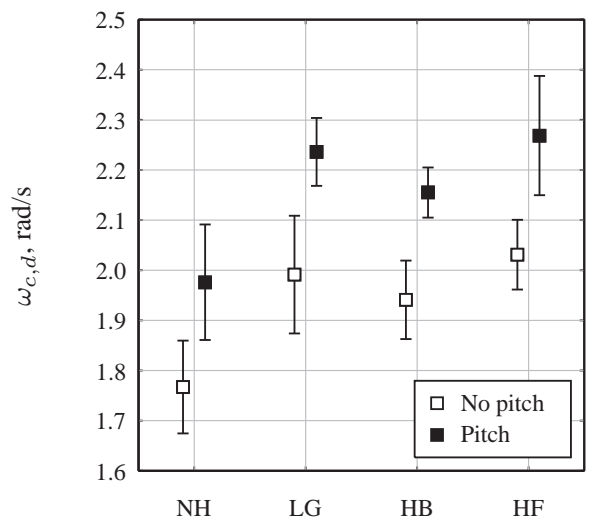

(c) Disturbance phase margin

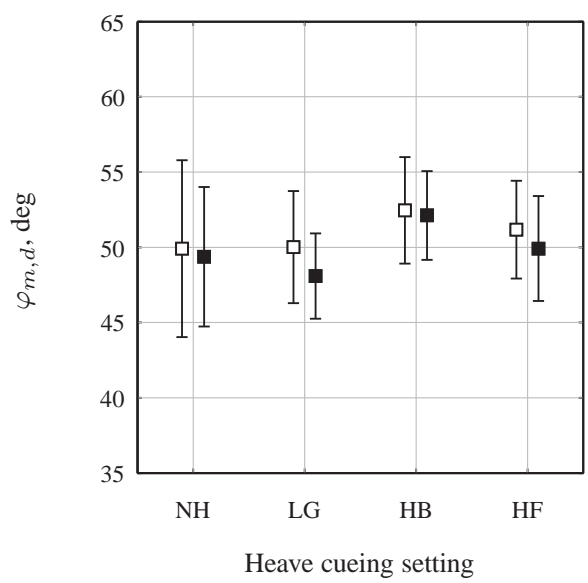

(b) Target crossover frequency

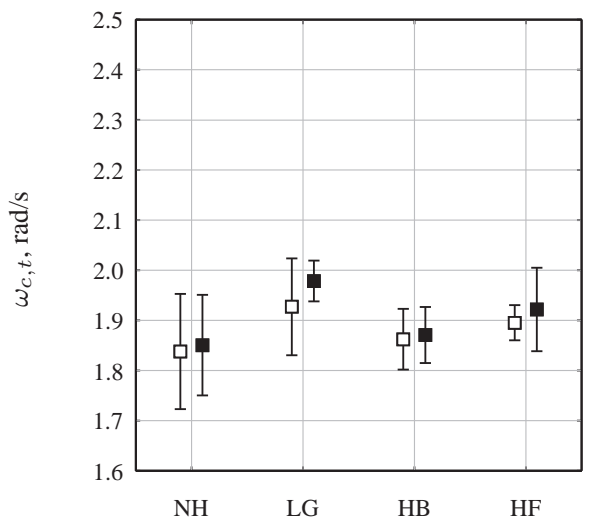

(d) Target phase margin

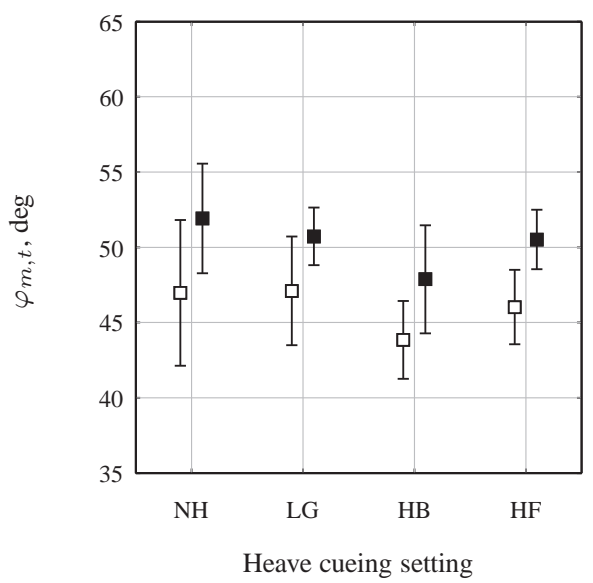

Figure 11. Average pilot-vehicle system crossover frequencies and phase margins.

Table 5. Within-subject ANOVA results for measured crossover frequencies and phase margins.

\begin{tabular}{|c|c|c|c|c|c|c|c|c|c|c|c|c|}
\hline \multirow{2}{*}{$\begin{array}{l}\begin{array}{l}\text { Independent } \\
\text { variables }\end{array} \\
\text { Factor }\end{array}$} & \multicolumn{12}{|c|}{ Dependent measures } \\
\hline & df & $\mathrm{F}$ & Sig. & df & $\mathrm{F}$ & Sig. & df & $\mathrm{F}$ & Sig. & $\mathrm{df}$ & $\mathrm{F}$ & Sig. \\
\hline pitch & 1,7 & 57.35 & $* *$ & 1,7 & 0.76 & - & 1,7 & 0.35 & - & 1,7 & 5.68 & $* *$ \\
\hline heave $\times$ pitch & 3,21 & 0.10 & - & 3,21 & 0.33 & - & 3,21 & 0.24 & - & 3,21 & 0.33 & - \\
\hline
\end{tabular}


The measured disturbance-loop phase margins presented in Fig. 11c show values of $\varphi_{m, d}$ of around 50 deg for all conditions of the experiment. Except perhaps for slightly elevated values (around 2 deg higher) for the HB heave motion filter setting, no consistent effects of the variation in pitch and heave cueing are observed on $\varphi_{m, d}$. This is also confirmed from the ANOVA results shown in Table 5.

The crossover frequencies of the target-following loop show a trend over the different heave cueing conditions that follows the trend observed in $\omega_{c, d}$, but markedly less pronounced. In addition, no consistent effect of the addition of rotational pitch cueing is present for $\omega_{c, t}$. As can be verified from Table 5, the observed variation in target crossover frequency due to heave cueing variations is statistically significant, while the ANOVA also confirms the absence of an effect of rotational pitch cueing.

The corresponding target-loop phase margin results presented in Fig. 11d do show a clear effect of the presence of rotational pitch cueing, as the addition of pitch cueing is seen to result in a consistent increase in $\varphi_{m, t}$ of around $5 \mathrm{deg}$ for all heave cueing settings. This increase in target phase margin is highly significant, as can be verified from Table 5 . A slight decrease in $\varphi_{m, t}$ is visible for the HB heave cueing setting, but overall no consistent effects of the variation in heave cueing on the target-loop phase margin are observed.

\section{V.C. Pilot Modeling Results}

\section{V.C.1. Pilot Model Structure Selection}

As depicted in Fig. 2, for the considered pitch attitude control task pilots may utilize a feedback control strategy based on information taken from visual tracking error and physical pitch and heave motion cues. For modeling pilots' responses to visually presented tracking errors $e$, the pilot visual response to perceived tracking errors $H_{p_{e}}(j \omega)$ was modeled as:

$$
H_{p_{e}}(j \omega)=K_{p_{v}}\left(T_{L_{v}} j \omega+1\right) e^{-j \omega \tau_{v}} H_{n m}(j \omega)
$$

The model given by Eq. (17) is based on McRuer et al.'s Precision Model for single-loop compensatory tracking. ${ }^{23}$ Note that the visual equalization term of the Precision Model is reduced to a pure lead, as for the controlled element dynamics given by Eq. (3) it is likely that pilots will generate lead to compensate for the second-order dynamics at frequencies above $\omega_{s p}$. The main parameters of the model for $H_{p_{e}}(j \omega)$ are the pilot visual gain $K_{p_{v}}$, the pilot visual lead time constant $T_{L_{v}}$, and the pilot visual delay $\tau_{v}$. The high-frequency neuromuscular actuation dynamics $H_{n m}(j \omega)$ are modeled using the same second-order mass-spring-damper model used in Refs. 5 and 6:

$$
H_{n m}(j \omega)=\frac{\omega_{n m}^{2}}{(j \omega)^{2}+2 \zeta_{n m} \omega_{n m} j \omega+\omega_{n m}^{2}}
$$

The neuromuscular actuation dynamics natural frequency $\omega_{n m}$ and damping factor $\zeta_{n m}$ are also considered as free parameters of the considered pilot model.

For modeling pilots' responses to perceived rotational pitch and translational heave accelerations an approach similar to the one described in Ref. 6 is followed. As for instance proposed by Hosman ${ }^{14}$ and Van der Vaart, ${ }^{15}$ pilots' responses to rotational pitch motion can be modeled as a response to the output of the semicircular canals (SCC). The dynamics of the SCC are approximately those of an integrator for a rotational acceleration input over the frequency range approximately $0.1-9 \mathrm{rad} / \mathrm{s},{ }^{14}$ after which they level of to a pure gain. Similarly, the otoliths, which are sensitive to specific force and are hence the vestibular sensor sensitive to the heave accelerations applied in the experiment, are approximately a gain over the frequency range of interest to manual control. However, it has been argued, ${ }^{31}$ and shown using experimental data in Ref. 6 , that in some instances pilots may internally integrate perceived translational accelerations to rates, to end up with an alternative for visual lead equalization.

For modeling pilots' responses to heave acceleration cues, a number of different strategies were evaluated. First of all, by evaluating pilot describing function estimates, obtained with a method similar to the one described in Ref. 13, it was found that pilot responses to heave motion only showed consistency at frequencies well above $\omega_{s p}=1.03 \mathrm{rad} / \mathrm{s}$ (this will be addressed in more detail below). As can be verified from Fig. 7, the PH acceleration cues are dominant at these higher frequencies, whereas the $\mathrm{CH}$ accelerations dominate below $\omega_{s p}$. Ref. 5 showed that $\mathrm{CH}$ cues hold no information that would be useful to pilots during pitch attitude control, which is confirmed by the purely highfrequency response to heave motion found in the current experiment. Based on this analysis it was found valid to model pilots' responses to heave accelerations $H_{p_{a_{z}}}(j \omega)$ using only the IPH accelerations $a_{z_{\theta}, i}$, rather than the total aircraft heave at the pilot station $a_{z}$, as given by Eq. (1).

The resulting models for the pilot pitch and heave responses as used in this paper are similar to those derived and applied in Ref. 6 and are given by: 


$$
\begin{aligned}
H_{p_{\theta}}(j \omega) & =K_{p_{m}} j \omega\left(T_{L_{m}} j \omega+1\right) e^{-j \omega \tau_{m}} H_{n m}(j \omega) \\
H_{p_{a_{z}}}(j \omega) & =\frac{-K_{p_{m}}}{l_{x_{p i l}}} \frac{T_{L_{m}} j \omega+1}{j \omega} e^{-j \omega \tau_{m}} H_{n m}(j \omega)
\end{aligned}
$$

In addition to the pilot motion gain $K_{p_{m}}$ and the pilot motion delay $\tau_{m}$, these models for the pilot pitch and heave responses include an additional parameter, the motion lead time constant $T_{L_{m}}$. This lead term is included in both models to allow for capturing of possible pilot responses to pitch and heave accelerations, instead of purely modeling the response to integrated heave acceleration, as for instance found appropriate for the experiment of Ref. 6.

As the pitch heave accelerations that are used as the input for the pilot heave response given by $H_{p_{a_{z}}}(j \omega)$ are related to the pitch attitude through $a_{z_{\theta}, i}=-l_{x_{p i l}} \ddot{\theta}$, this implies that the pilot model responses to rotational pitch cues and heave acceleration cues should yield equivalent model fits if both motion cues are utilized by pilots to the same extent. This, as also argued in Ref. 6, allows for direct comparison of values of $K_{p_{m}}$ and $\tau_{m}$ across the different model forms given by Eqs. (19) and (21).

Fig. 12 depicts pilot model identification results for one subject for one of the experimental conditions with only heave motion cueing (subject 1, condition HF). For reference, Fig. 12 also shows an estimated frequency-domain pilot describing function for this data set, which can be estimated at the forcing function frequencies independent from the chosen pilot model structure. A fit of the model of Eq. (21), obtained using the time-domain identification technique detailed in Ref. 17, is also depicted (solid black lines). Note that the fitted model, which incorporates both the pilot visual and heave responses (Eqs. (17) and (21), respectively), describes $86 \%$ of the variance in the measured control signal $u$, as indicated by the pilot model VAF.

In addition to the fitted pilot model, also the pilot heave response is depicted where the lead time constant $T_{L_{m}}$ is set to zero. Comparison with the independently measured describing function suggests pilots were responding to acceleration information at the highest measurement frequencies, and therefore confirms the importance of including this extra lead term in the model for $H_{p_{a_{z}}}(j \omega)$.

The use of two forcing functions in a compensatory tracking task has been shown to allow for reliable separation of pilots' responses to two different modalities. ${ }^{13}$ Therefore, for this experiment is was not possible to estimate both the pilot responses to rotational pitch and translational heave motion, $H_{p_{\theta}}(j \omega)$ and $H_{p_{a z}}(j \omega)$, respectively. Due to the equivalence of the models given by Eqs. (19) and (eq:Hpaz), this was not a problem for evaluating the contributions of the visual and vestibular modalities, however, it does imply no explicit distinction can be made between behavior driven by rotational pitch motion and translational heave motion cues. Furthermore, it should be noted that for the condition without pitch and heave motion cueing, only the visual pilot response as given by Eq. (17) was fitted to measurements of $e$ and $u$.

\section{V.C.2. Pilot Model Parameter Estimates}

Fig. 13 presents the average identified parameters of the model for the pilot visual response $H_{p_{e}}(j \omega)$, as given by Eq. (17). Table 6 shows the corresponding ANOVA results. For the pilot visual gain $K_{p_{v}}$, Fig. 13a shows an increasing trend with increasing heave motion, which is especially clear for the conditions without additional rotational pitch cueing (white markers). Furthermore, a slight drop in visual gain appears to be present for the HB and HF conditions if rotational pitch motion is made available. However, as can be judged from Table 6, none of these observations are statistically significant.

Fig. 13b shows the variation in the pilot visual lead time constant $T_{L_{v}}$ over the different conditions of the experiment. For this experiment, the addition of heave cueing in any form is seen to yield increased lead time constants compared to the NH conditions where no heave cueing was present. Typically, the availability of physical motion feedback is seen to yield a decrease in the amount of visual lead equalization performed by pilots during manual tracking. 5, 6, 12,14, 15 In Ref. 5, however, where a variation in the presence of $\mathrm{CH}$ and $\mathrm{PH}$ motion cues was considered for a pitch control task, a similar relative increase in $T_{L_{v}}$ was observed when $\mathrm{CH}$ motion was made available. In this experiment, always the full aircraft heave motion was presented, so including the $\mathrm{CH}$ motion component. Therefore, the increase in $T_{L_{v}}$ shown in Fig. 13b is consistent with the data from Ref. 5. The amount of visual lead generation is seen to increase even further when rotational pitch motion is made available. These effects of heave and pitch cueing variations on the pilot visual lead time constant are found to be highly significant and marginally significant, respectively (see Table 6). Furthermore, the increase in $T_{L_{v}}$ for conditions HB and HF compared to NH and LG is found 
(a) Visual magnitude

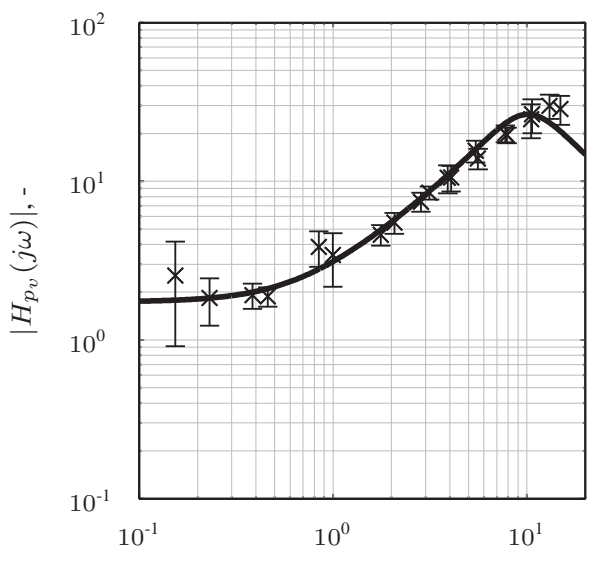

(c) Visual phase

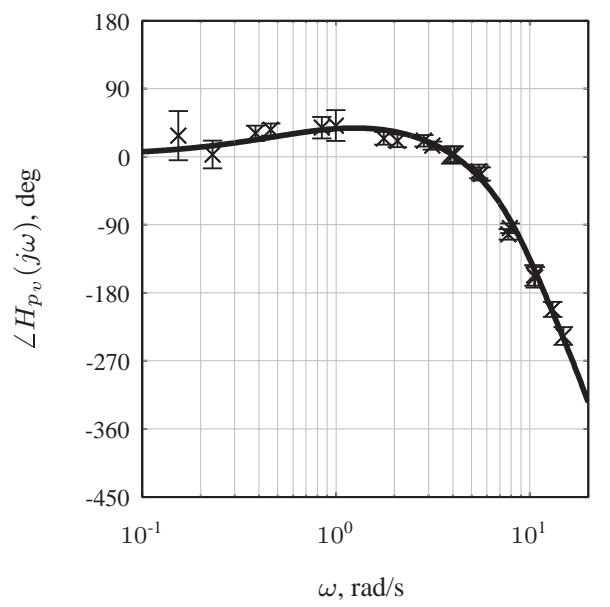

(b) Motion magnitude

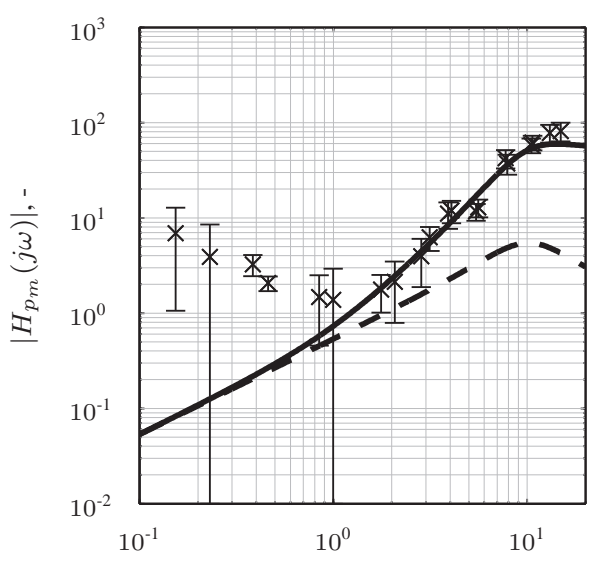

(d) Motion phase

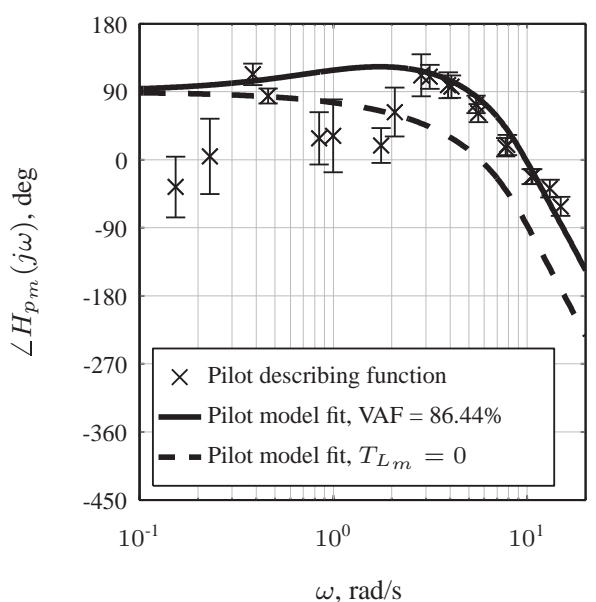

Figure 12. Example of pilot visual and heave responses according to Eqs. (17) and (21) fit to measured data (subject 1, condition HF). 
(a) Visual gain

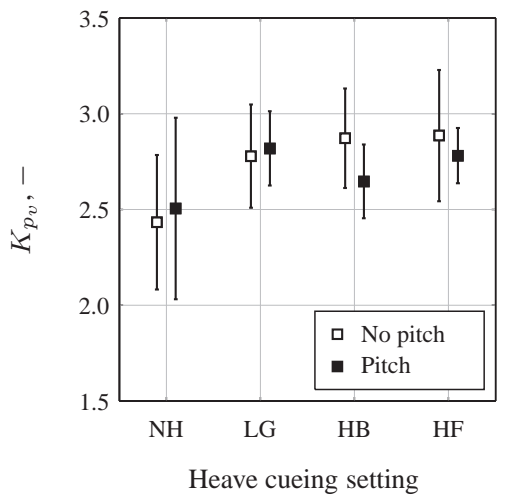

(b) Visual lead constant

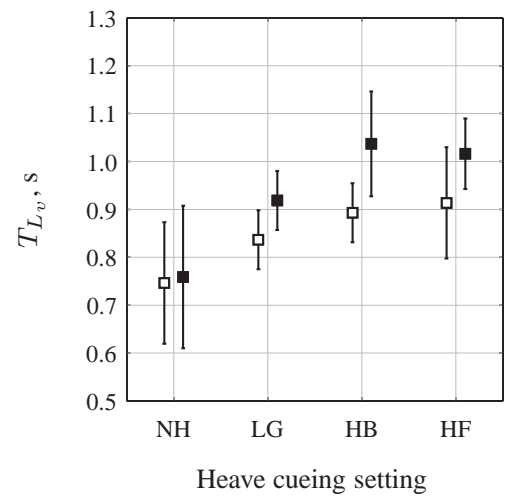

(c) Visual delay

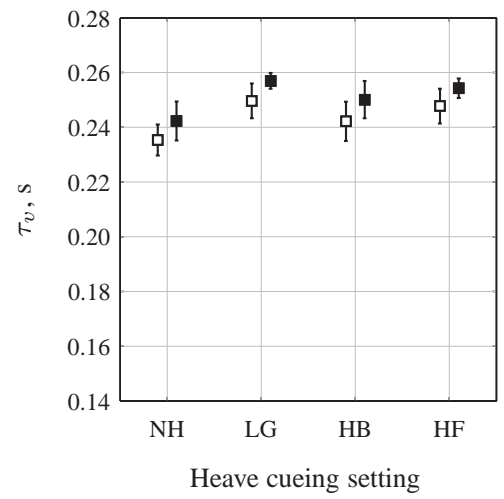

Figure 13. Average pilot model visual response parameters.

Table 6. Within-subject ANOVA results for visual channel model parameters.

\begin{tabular}{|c|c|c|c|c|c|c|c|c|c|}
\hline \multirow{3}{*}{$\begin{array}{l}\begin{array}{l}\text { Independent } \\
\text { variables }\end{array} \\
\text { Factor }\end{array}$} & \multicolumn{9}{|c|}{ Dependent measures } \\
\hline & \multicolumn{3}{|c|}{$K_{p_{v}}$} & \multicolumn{3}{|c|}{$T_{L_{v}}$} & \multicolumn{3}{|c|}{$\tau_{v}$} \\
\hline & $\mathrm{df}$ & $\mathrm{F}$ & Sig. & $\mathrm{df}$ & $\mathrm{F}$ & Sig. & $\mathrm{df}$ & $\mathrm{F}$ & Sig. \\
\hline heave & $1.11,7.77^{a}$ & 1.93 & - & $1.26,8.83^{a}$ & 5.87 & $* *$ & 3,21 & 8.11 & $* *$ \\
\hline pitch & 1,7 & 0.37 & - & 1,7 & 4.51 & $*$ & 1,7 & 14.20 & $* *$ \\
\hline heave $\times$ pitch & 3,21 & 1.21 & - & 3,21 & 4.76 & $* *$ & 3,21 & 0.04 & - \\
\hline & $\begin{aligned} * * & = \\
* & = \\
- & = \\
a & =\end{aligned}$ & $\begin{array}{l}\text { highly s } \\
\text { margina } \\
\text { not sign } \\
\text { Greenh }\end{array}$ & $\begin{array}{l}\text { nificar } \\
\text { signi } \\
\text { cant ( } \\
\text { se-Gei }\end{array}$ & $\begin{array}{l}(p<0.05) \\
\text { cant }(0.05 \leq \\
\geq 0.1) \\
\text { er sphericity }\end{array}$ & $<0$ & applic & & & \\
\hline
\end{tabular}

to be larger in the presence of pitch motion than for the pure heave cueing conditions, yielding a highly significant interaction between pitch and heave motion variation, as can be judged from Table 6 .

The pilot visual delay $\tau_{v}$ is seen to show a consistent increase of around $0.01 \mathrm{sec}$ when rotational pitch motion cues are made available, independent of differences in heave cueing (see Fig. 13c). This increase in $\tau_{v}$ with the presence of pitch cueing is found to a highly significant effect, as can be verified from the right-most columns in Table 6. In addition, identified values of the visual delay parameter also show considerable variation with the applied differences in heave cueing. Compared to the $\mathrm{NH}$ condition, pilot visual delays are found to be around $0.02 \mathrm{sec}$ higher for heave cueing filter settings LG and HF. For the HB filter, the increase in $\tau_{v}$ compared to NH is markedly less. This effect of heave motion cueing on $\tau_{v}$ is also found to be statistically significant (see Table 6). Previous studies have shown increased visual delays with increasing levels of motion fidelity $5,6,12$ and the identified values from the current experiment are consistent with these previous findings.

Fig. 14 presents the identified values of the pilot motion response dynamics as given by Eqs. (19) or (21). Due to the absence of values for these three parameters for the condition with no heave and no pitch cueing $(\mathrm{NH})$ an ANOVA as performed on all other dependent measures could not be performed on this data. Therefore, only the variations in $K_{p_{m}}, T_{L_{m}}$, and $\tau_{v}$ over the other three heave conditions (LG, HB, and HF) were analyzed using an ANOVA with one less level of the "heave" independent variable. The results of this ANOVA are shown in Table 7.

Identified values of the pilot motion gain are presented in Fig. 14a. Note that higher values of $K_{p_{m}}$ do not directly correspond to increased reliance on motion feedback due to different magnitude and phase distortion of the signal that drives the pilot motion response for the different heave cueing settings $\left(a_{z_{s}}\right.$, see Fig. 2). The relative contributions of the visual and vestibular modalities to the pilot control strategy selected for the different conditions of the experiment are analyzed explicitly in Section V.C.3. Fig. 14a shows that the lowest values of $K_{p_{m}}$ are found for the condition with only rotational pitch cueing $(\mathrm{NH})$, for which the $95 \%$ confidence bars even include zero, suggesting only a minor effect of rotational pitch motion feedback on pilot control behavior for this condition.

Fig. 14a further shows a trend in $K_{p_{m}}$ that is consistent with the variation in motion filter gain over the different conditions. The highest pilot motion gains are observed for the LG filter, which has the lowest $K_{h p}$ of 0.3 , while the lowest $K_{p_{m}}$ are found for the HB filter for which $K_{h p}=0.7$. Note, however, that especially for the conditions without pitch motion the changes in $K_{p_{m}}$ are not of the same magnitude as those in $K_{h p}$, suggesting varying usage of motion information over the different experimental conditions. As visible from the large $95 \%$ confidence interval for the data obtained for the LG filter with pitch motion, for one subject an outlier, that is, a comparatively large value of $K_{p_{m}}$ of 
(a) Motion gain

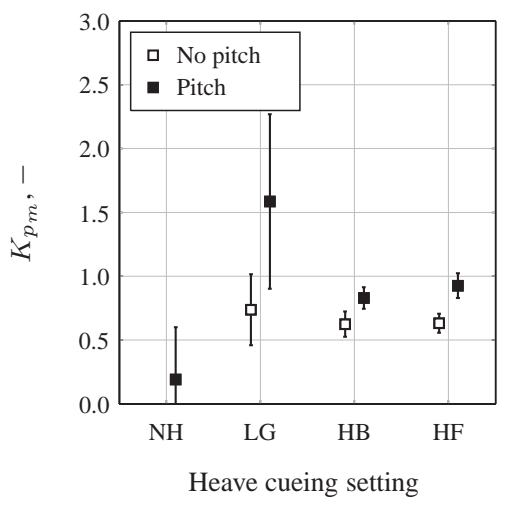

(b) Motion lead constant

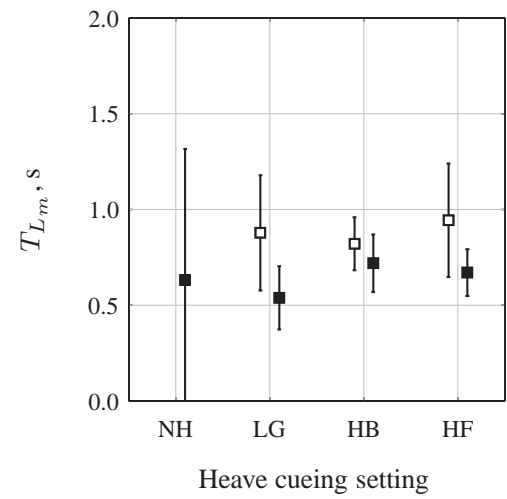

(c) Motion delay

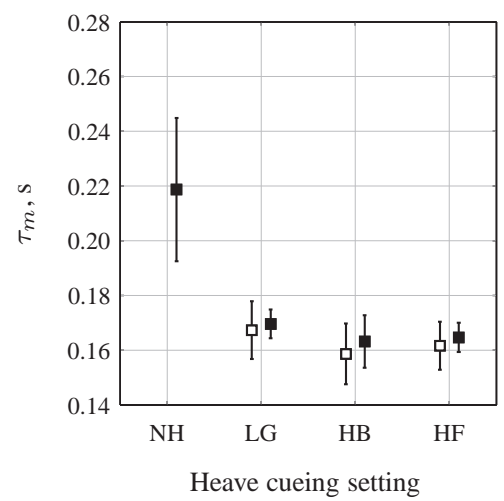

Figure 14. Average pilot model motion response parameters.

Table 7. Within-subject ANOVA results for motion channel model parameters.

\begin{tabular}{|c|c|c|c|c|c|c|c|c|c|}
\hline \multirow{3}{*}{$\begin{array}{l}\text { Independent } \\
\text { variables } \\
\text { Factor }\end{array}$} & \multicolumn{9}{|c|}{ Dependent measures } \\
\hline & \multicolumn{3}{|c|}{$K_{p_{m}}$} & \multicolumn{3}{|c|}{$T_{L_{m}}$} & \multicolumn{3}{|c|}{$\tau_{m}$} \\
\hline & df & $\mathrm{F}$ & Sig. & $\mathrm{df}$ & $\mathrm{F}$ & Sig. & df & $\mathrm{F}$ & Sig. \\
\hline heave & $1.04,7.27^{a}$ & 13.34 & $* *$ & 2,14 & 0.58 & - & $1.13,7.88^{a}$ & 1.50 & - \\
\hline pitch & 1,7 & 8.26 & $* *$ & 1,7 & 7.61 & $* *$ & 1,7 & 1.46 & - \\
\hline \multirow[t]{5}{*}{ heave $\times$ pitch } & $1.04,7.30^{a}$ & 2.71 & - & 2,14 & 0.80 & - & 2,14 & 0.12 & - \\
\hline & $* *$ & \multicolumn{8}{|c|}{ highly significant $(p<0.05)$} \\
\hline & $=$ & \multicolumn{8}{|c|}{ marginally significant $(0.05 \leq p<0.1)$} \\
\hline & - & \multicolumn{8}{|c|}{ not significant $(p \geq 0.1)$} \\
\hline & $=$ & \multicolumn{8}{|c|}{ Greenhouse-Geisser sphericity correction applied } \\
\hline
\end{tabular}

4.50, was found for this condition. Without the data for this one subject the average $K_{p_{m}}$ for this condition would be 1.17 , so still the highest measured value of all conditions, instead of the presented 1.59 average. Finally, note from Fig. 14a that motion gains are found to be consistently higher for those conditions for which both pitch and heave cueing were available than for those with pure heave cueing. As can be verified from Table 7, both the effects of pitch and heave cueing on $K_{p_{m}}$ are found to be highly significant.

The identified values of the motion lead time constant $T_{L_{m}}$ are shown in Fig. 14b. Again, the large spread observed for the estimates of $T_{L_{m}}$ for the NH condition are the result of a single outlier in the identified parameters. For one subject a value for the motion lead time constant of $2.64 \mathrm{~s}$ was found for this condition, whereas for the other seven subjects $T_{L_{m}}$ was found to be $0.34 \pm 0.082 \mathrm{~s}$ (mean $\pm 95 \%$ confidence interval). When considering the heave cueing conditions LG, HB, and HF, only an effect of the presence of rotational pitch cueing on the motion lead time constant is observed, as can be verified from Table 7. For conditions with both pitch and heave cueing $T_{L_{m}}$ is found to be around $0.64 \mathrm{sec}$, while time constants of on average $0.88 \mathrm{sec}$ are found for the conditions with only heave cueing. This decrease in $T_{L_{m}}$ with the addition of pitch motion, which indicates a reduction in the usage of acceleration feedback and a pilot response based on rate feedback over a wider frequency range, is a highly significant effect. As $T_{L_{m}}$ was also generally found to be markedly lower for the NH condition, where only rotational pitch motion was present, this reduction in $T_{L_{m}}$ suggests a control strategy in which feedback from rotational information perceived with the semicircular canals is used in addition to perceived heave cues. This, however, is not proven by the presented data due to the fact that only the combined response to pitch and heave motion cues is modeled here (see Section V.C.1).

Fig. $14 \mathrm{c}$ shows the measured values of the pilot motion delay $\tau_{m}$. As can be verified from Fig. $14 \mathrm{c}$ and Table 7 , $\tau_{m}$ is found to be largely invariant with the applied variation in pitch and heave cueing settings. Slightly higher values of the pilot motion delay are found for the LG heave cueing condition compared to HB and HF, but this is not a significant effect. A markedly higher average $\tau_{m}$ (around $0.05 \mathrm{sec}$ higher) is, however, found for the condition with only pitch cueing $(\mathrm{NH})$. This indicates that for the $\mathrm{NH}$ condition, for which the identified motion gains depicted in Fig. 14a indicate only a very limited effect on pilot behavior, also the latency in the pilot motion response channel was markedly higher. A similar difference in pilot motion delay between conditions with only pitch cueing and those with additional heave cueing was reported in Ref. 6.

Fig. 15 shows the identified parameters of the neuromuscular actuation model as defined by Eq. (18). For the neuromuscular actuation natural frequency $\omega_{n m}$ (see Fig. 15a), a clear variation over the different heave cueing settings is observed. For the heave motion filters with the highest gains, values of the neuromuscular frequency are found to be 
(a) Neuromuscular frequency

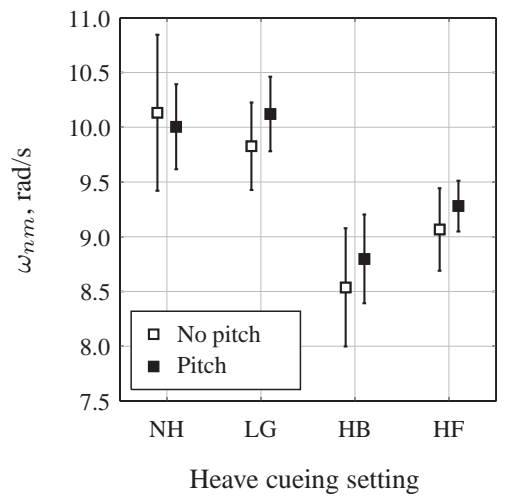

(b) Neuromuscular damping

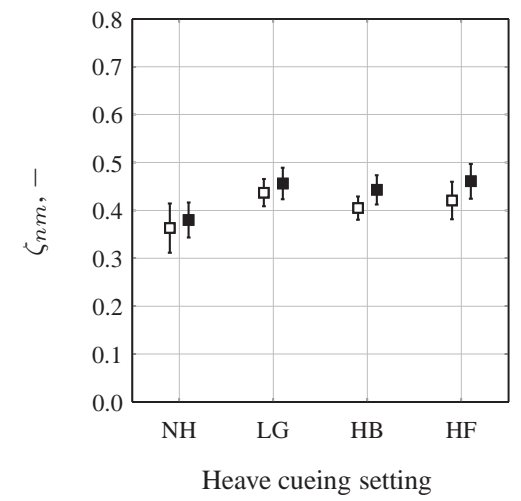

Figure 15. Average pilot neuromuscular actuation model parameters.

Table 8. Within-subject ANOVA results for neuromuscular model parameters.

\begin{tabular}{lcccccc}
\hline \hline \multirow{2}{*}{$\begin{array}{l}\text { Independent } \\
\text { variables }\end{array}$} & \multicolumn{6}{c}{ Dependent measures } \\
\cline { 2 - 7 } & $\mathrm{df}$ & $\mathrm{F}$ & Sig. & $\mathrm{df}$ & $\mathrm{F}$ & Sig. \\
\hline Factor & $1.65,11.57^{a}$ & 14.89 & $* *$ & 3,21 & 5.88 & $* *$ \\
\hline heave & 1,7 & 2.44 & - & 1,7 & 3.94 & $*$ \\
pitch & 3,21 & 0.69 & - & 3,21 & 0.96 & - \\
heave $\times$ pitch & &
\end{tabular}

$* * \quad=\quad$ highly significant $(p<0.05)$

$* \quad=$ marginally significant $(0.05 \leq p<0.1)$

$-\quad=\quad$ not significant $(p \geq 0.1)$

$a=$ Greenhouse-Geisser sphericity correction applied

around $1 \mathrm{rad} / \mathrm{s}$ lower than those observed for NH and LG. Note that the decrease is largest for the HB condition, which has the highest high-frequency gain. No clear effect of the presence of rotational pitch motion is found for $\omega_{n m}$. Both these observations from Fig. 15a are confirmed by the ANOVA results presented at left in Table 8, which show a only a highly significant effect of the variation in heave cueing.

For the neuromuscular actuation damping ratio $\zeta_{n m}$, Fig. $15 \mathrm{~b}$ shows trends with the applied variation in pitch and heave motion cueing that are comparable to those shown for $\tau_{v}$ in Fig. 13c. The same increase for conditions with heave motion as also found for the pilot visual delay is also observed for $\zeta_{n m}$, including the reduced increase for the HB conditions. Though less consistent than found for $\tau_{v}$, the identified values of $\zeta_{n m}$ are also found to increase slightly when pitch motion is available. As listed in Table 8 , the effects of the variations in heave and pitch cueing on $\zeta_{n m}$ are found to be highly and marginally significant, respectively.

\section{V.C.3. Quality of Fit and Motion Feedback Contribution}

Fig. 16a shows the average model VAF for the fitted pilot models for all experimental conditions. The corresponding ANOVA results are presented in Table 9. On average, the VAF values are found to be between 87 and $92 \%$ for all conditions, indicating that the fitted pilot models are able to describe the measured data with high accuracy. As can be verified from Fig. 16a, pilot model VAFs are found to be lowest for the HB and HF filter settings, both for the conditions with and without additional rotational pitch cueing. Though this decrease in model VAF is modest, not even 5\% compared to the $\mathrm{NH}$ conditions, it is found to be a statistically significant effect (see Table 9). This slight decrease in VAF could either result from the fact that the chosen model is less appropriate for the data from these conditions, or could be caused by reduced linearity of pilot behavior under these cueing conditions. The ANOVA further revealed a marginally significant interaction between the effects of pitch and heave cueing, which post-hoc analysis revealed to be caused by the differences in the pilot model VAF between the NH and LG conditions: without pitch motion the VAFs for both heave cueing settings are found to be approximately equal, while with pitch cueing the LG filter is found to yield a clearly lower VAF than observed for $\mathrm{NH}$.

Fig. 16b depicts the fraction between the variances of the control signal contributions of the pilot visual $\left(u_{v}\right)$ and the pilot motion $\left(u_{m}\right)$ responses, see Fig. 2. The corresponding ANOVA results are presented in Table 9 at right. Fig. 16b shows an increasing trend in $\sigma_{u_{m}}^{2} / \sigma_{u_{v}}^{2}$ over the different heave motion settings, reaching its highest values for the HB filter. Furthermore, the presented data show a consistent increase of around 0.05 due to the addition of 
(a) Variance Accounted For

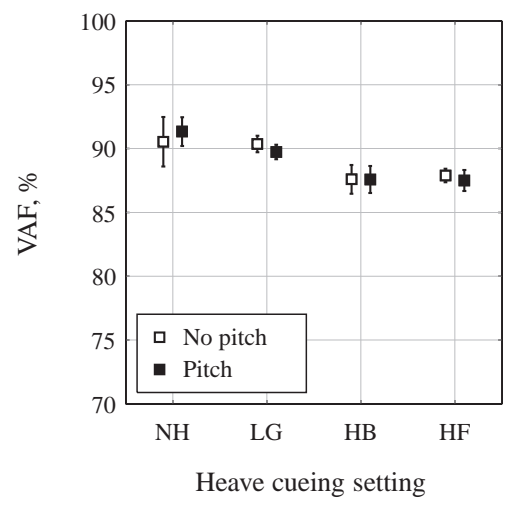

(b) Control variance fraction

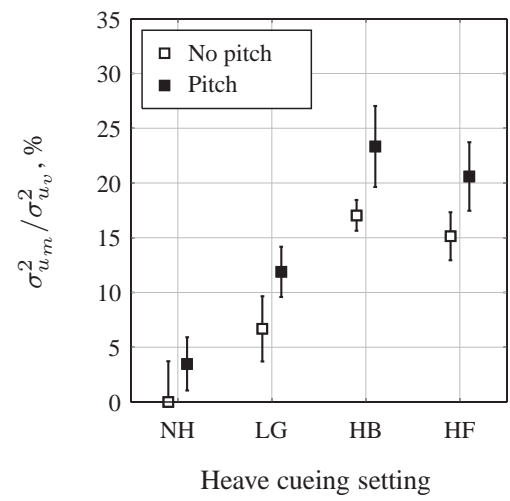

Figure 16. Mean pilot model variance accounted for for all conditions.

Table 9. Within-subject ANOVA results for variance accounted for and control signal variances.

\begin{tabular}{|c|c|c|c|c|c|c|c|}
\hline \multirow{2}{*}{\multicolumn{2}{|c|}{$\begin{array}{l}\text { Independent } \\
\text { variables }\end{array}$}} & \multicolumn{6}{|c|}{ Dependent measures } \\
\hline & & \multicolumn{3}{|c|}{ VAF } & \multicolumn{3}{|c|}{$\sigma_{m}^{2} / \sigma_{v}^{2}$} \\
\hline \multicolumn{2}{|l|}{ Factor } & $\mathrm{df}$ & $\mathrm{F}$ & Sig. & $\mathrm{df}$ & $\mathrm{F}$ & Sig \\
\hline \multicolumn{2}{|l|}{ heave } & $1.30,9.14^{a}$ & 13.22 & $* *$ & $1.30,9.09^{a}$ & 71.33 & $* *$ \\
\hline \multicolumn{2}{|l|}{ pitch } & 1,7 & 0.05 & - & 1,7 & 11.27 & $* *$ \\
\hline \multicolumn{2}{|l|}{ heave $\times$ pitch } & 3,21 & 2.88 & $*$ & 3,21 & 2.46 & * \\
\hline$* *$ & $=$ & highly signif & ant $(p<$ & $0.05)$ & & & \\
\hline$*$ & $=$ & marginally si & nificant & $.05 \leq$ & $p<0.1)$ & & \\
\hline- & $=$ & not significar & $(p \geq 0$ & & & & \\
\hline$a$ & $=$ & Greenhouse- & jeisser $\mathrm{sp}$ & ericity & orrection app & & \\
\hline
\end{tabular}

rotational pitch motion. As can be verified from Table 9, both these effects are found to be statistically significant. The marginally significant interaction between the variation in pitch and heave cueing listed in Table 9 was found to result mainly from the smaller difference between the pitch and no-pitch conditions for the NH filter compared to the other heave motion filter settings. Overall, Fig. 16b demonstrates marked differences in the contribution of motion feedback to pilot control inputs as a result of the applied variation in pitch and heave cueing. Especially the relatively low $\sigma_{u_{m}}^{2} / \sigma_{u_{v}}^{2}$ for the LG conditions and the fact that the highest values are found for the HB filter conditions are notable, and are consistent with the pilot model parameter estimates shown in Fig. 13 to Fig. 15.

\section{Discussion}

This paper describes an effort to evaluate the effect of varying pitch and heave motion cueing on pilot tracking performance and control behavior in a pitch attitude disturbance-rejection task for a large commercial airliner. An extensive analysis of aircraft geometry and dynamics was performed to evaluate to what extent earlier findings from studies that considered much smaller aircraft ${ }^{5,6}$ might be directly applicable. The most important factor that might prevent his was thought to be the much larger heave accelerations at the pilot station due to aircraft pitch accelerations $(\mathrm{PH})$, as the distance between the center of pitch rotation and the pilot station is markedly larger for large transport aircraft. An experiment is described in which eight subjects performed the pitch tracking task for varying motion cueing settings. In the experiment, three different heave washout filters were evaluated in addition to a reference condition without any heave motion cueing. The resulting four heave cueing conditions were also performed with and without pitch rotation, resulting in a total of eight experimental conditions.

The performed experiment showed significantly better tracking performance for the heave motion filter settings with higher motion filter gains. In addition, despite the suspected dominance of the heave motion cues at the pilot station, a further significant increase in tracking performance was still observed with the addition of rotational pitch motion cues. The effect of rotational pitch motion cues on pilot tracking performance was, however, found to be less pronounced than reported for smaller aircraft in Refs. 5 and 6. Measured disturbance-loop crossover frequencies were found to reflect the same trend with the applied variation in pitch and heave cueing as observed in the tracking error variance. Disturbance crossover frequencies were found to increase around $0.2 \mathrm{rad} / \mathrm{s}$ with the addition of rotational pitch motion, and with $0.3 \mathrm{rad} / \mathrm{s}$ for the highest fidelity motion filter (HF) compared to the no heave cueing condition 
(NH). As also reported by Pool et al., ${ }^{6}$ the addition of rotational pitch motion was further found to result in a significant increase in target-loop phase margin, independent of the heave motion cueing setting.

The dominant effects on pilot behavior of the applied variation in pitch and heave motion cueing that were determined using fitted multimodal pilot models include a significant increase in the pilot visual lead time constant and the pilot visual time delay for the two heave filter settings with the highest motion filter gains (HF and HB) and with the addition of rotational pitch motion cues. The pitch cues are also found to result in significantly higher pilot motion gains for all heave cueing settings. Especially the increase in the visual lead time constant seems surprising, as it is commonly found that the availability of motion feedback allows pilots to reduce the amount of visual lead equalization. ${ }^{6,12,14,15}$ The same opposite effect was also observed by Zaal et al. ${ }^{5}$ for those conditions of their experiment in which the heave motion resulting from changes in vertical aircraft c.g. position $(\mathrm{CH})$ was present. The experiment of Ref. 6 confirmed that this increase in visual lead equalization indeed resulted from the presence of the $\mathrm{CH}$ cues. The portion of the pilot control signal explained by the pilot motion response, however, was found to be highest for those conditions that also showed the highest values for the visual lead time constant, implying still increased use was made of the available motion feedback, as expected. Furthermore, the LG heave cueing setting, which used a comparatively low-gain low-break frequency high-pass filter, was found to yield pilot tracking behavior that was closest to that observed without heave cueing, as for instance visible from a relatively modest increase in the visual lead time constant.

Pilot describing function and pilot model analysis showed pilots were responding to perceived PH heave accelerations directly at frequencies above 1-2 rad/s - that is, above the motion filter break frequencies that were evaluated in this experiment - rather than integrating them to rates to alleviate the required visual lead equalization as found in previous studies. ${ }^{6,31}$ An optimal control analysis performed to assess the difference in the importance of attitude, rate, and acceleration feedback for accurate tracking with the considered Boeing 747 pitch dynamics and the Cessna Citation I dynamics considered in earlier experiments ${ }^{5,6}$ had predicted increased benefit of acceleration feedback for the comparatively slow Boeing 747 dynamics.

The heave motion filter settings that were evaluated in the experiment were chosen to reflect different choices in the inevitable trade-off between low motion filter phase distortion and high motion filter gain. It must be noted that all selected filters would still be considered low fidelity according to the fidelity criterion proposed by Schroeder. ${ }^{10}$ This is a result of the large-amplitude low-frequency heave motions that inevitably result from aircraft pitch maneuvering, and which are almost impossible to replicate with high motion fidelity on a typical flight simulator. ${ }^{2,3,18,20}$ As also stated by Schroeder and Grant, ${ }^{11}$ the value of having reference measurements of pilot behavior for a condition where heave motion is presented 1-to-1 (no washout), which would allow for evaluation of the severity of this problem in flight simulator heave motion cueing, cannot be overstated. The fact that this study, however, already shows significant adaptation of pilot behavior even for a variation in low-fidelity heave motion suggests behavioral differences with measurements taken under real-flight heave cueing conditions may be appreciable.

Furthermore, one important issue that is not explicitly evaluated in this paper is the coordination between the cueing of the different degrees of freedom that are importance during pitch maneuvering. Due to the fact that typically a lot less attenuation is required for cueing rotational degrees of freedom than for translational motion cueing, discrepancies may result between these cues in flight simulators. The same, of course, holds for filtered motion cues that are presented in simulators in parallel to unfiltered visual cues. For the experiment considered in this paper, where pitch motion cues (and visual cues) were always presented 1-to-1, the largest discrepancies between the different cues occurred with the HB and HF motion filters, which had the highest break frequencies of all considered heave motion cueing settings, 1.25 and $0.85 \mathrm{rad} / \mathrm{s}$, respectively. Despite recording the largest contributions of the pilot motion response to the given control inputs, measured data for these conditions do show values for some dependent measures that are consistent with degraded motion fidelity. Notable examples are a (non-significant) decrease in pilot visual gain, the highest recorded values for the pilot visual lead time constant, and a slight reduction in the pilot visual delay. Also the observed reduction in pilot model VAF for the conditions in which these two heave filter settings were used may be indicative of this reduced consistency in the perceived cues. Explicit evaluation of the effects of such a discrepancy between different related motion cues in flight simulators on pilots' use of these cues for manual control is therefore thought to be an important topic for future research.

Finally, the experiment described in this paper considered three different settings of a third-order high-pass heave motion filter, all with distinct values of the motion filter gain and break frequency. As identified by Sinacori, ${ }^{20}$ the attenuation performed by such a high-pass filter consists of motion scaling and phase distortion. It would be highly valuable to assess the effects of both these distinct operations on the supplied simulator motion by evaluating a factorial variation in motion filter gain and break frequency, with a large number of evaluated values for both, in an experiment similar to the one described in this paper. 


\section{Conclusions}

An experiment was performed to evaluate how pilot pitch attitude tracking behavior and performance are affected by a variation in rotational pitch and translational heave motion fidelity. The focus of the current paper was on manual control of a large commercial airliner (Boeing 747), for which both the controlled pitch dynamics and the relative magnitude of the perceivable pitch and heave motion cues are distinctly different from the smaller jet aircraft considered in previous studies into this problem. Analytical evaluation of differences in aircraft dynamics and geometry suggested increased requirement for rate and acceleration feedback, suggesting increased importance of motion feedback. Furthermore, comparatively larger effects of heave motion were expected than found in previous work, due to the increased magnitude of the heave cues perceivable at the pilot station, which result from the much larger aircraft body length. Pilot describing functions and multimodal pilot model analysis revealed pilots indeed used the cued heave accelerations for feedback at frequencies above 1-2 rad/s, instead of internally integrating perceived accelerations to allow for a rate feedback control strategy, as suggested in previous research. In addition, the contribution of motion feedback to the adopted pilot control strategy was found to be highest for the heave motion filter settings with the highest filter gains, which supplied the least attenuated high-frequency heave motion cues. The addition of rotational pitch motion was found to result in a further, though less pronounced compared to findings for smaller aircraft, increase in pilots' reliance on motion as opposed to visual feedback.

\section{Acknowledgments}

This research was supported by the Dutch Technology Foundation (STW), the applied science division of The Netherlands Organization for Scientific Research (NWO), and the technology program of the Ministry of Economic Affairs.

\section{References}

\footnotetext{
${ }^{1}$ Schmidt, S. F. and Conrad, B., "Motion Drive Signals for Piloted Flight Simulators," Tech. Rep. NASA CR-1601, National Aeronautics and Space Administration, Ames Research Center, 1970.

${ }^{2}$ Hosman, R. J. A. W., Van de Moesdijk, G. A. J., and Van der Vaart, J. C., "Optimalization and Evaluation of Linear Motion Filters," Fifteenth Annual Conference on Manual Control, Wright State University, Dayton (OH), 1979, pp. 213-242.

${ }^{3}$ Reid, L. D. and Nahon, M. A., "Flight Simulation Motion-Base Drive Algorithms. Part 1: Developing and Testing the Equations," Tech. Rep. UTIAS 296, University of Toronto, Institute for Aerospace Studies, Dec. 1985.

${ }^{4}$ Van Gool, M. F. C. and Mooij, H. A., "A Comparison of In-Flight and Ground-Based Pitch Attitude Tracking Experiments," Proceedings of the Twelfth Annual Conference on Manual Control, 1976, pp. 443-454.

${ }^{5}$ Zaal, P. M. T., Pool, D. M., De Bruin, J., Mulder, M., and Van Paassen, M. M., "Use of Pitch and Heave Motion Cues in a Pitch Control Task," Journal of Guidance, Control, and Dynamics, Vol. 32, No. 2, 2009, pp. 366-377.

${ }^{6}$ Pool, D. M., Zaal, P. M. T., Van Paassen, M. M., and Mulder, M., "Effects of Heave Washout Settings in Aircraft Pitch Disturbance Rejection," Journal of Guidance, Control, and Dynamics, Vol. 33, No. 1, 2010, pp. 29-41.

${ }^{7}$ Zaal, P. M. T., Pool, D. M., Van Paassen, M. M., and Mulder, M., "Comparing Multimodal Pilot Pitch Control Behavior Between a Simulator and Real Flight," AIAA Modeling and Simulation Technologies Conference and Exhibit, Portland (OR), August 8-11, 2011 (submitted for publication).

${ }^{8}$ Lombaerts, T. J. J., Smaili, M. H., Stroosma, O., Chu, Q. P., Mulder, J. A., and Joosten, D. A., "Piloted Simulator Evaluation Results of New Fault-Tolerant Flight Control Algorithm," Journal of Guidance, Control, and Dynamics, Vol. 32, No. 6, November/December 2009, pp. $1747-1765$.

${ }^{9}$ Groot, T., Damveld, H. J., Mulder, M., and Van Paassen, M. M., "Effects of Aeroelasticity on the Pilots Psychomotor Behavior," Proceedings of the AIAA Atmospheric Flight Mechanics Conference and Exhibit, Keystone, Colorado, Aug. 21-24, 2006, No. AIAA-2006-6494, American Institute of Aeronautics and Astronautics, Aug. 2006.

${ }^{10}$ Schroeder, J. A., "Helicopter Flight Simulation Motion Platform Requirements," Tech. Rep. NASA-TP-1999-208766, National Aeronautics and Space Administration, July 1999.

${ }^{11}$ Schroeder, J. A. and Grant, P. R., "Pilot Behavioral Observations in Motion Flight Simulation," Proceedings of the AIAA Guidance, Navigation, and Control Conference, Toronto, Canada, Aug. 2-5, No. AIAA-2010-8353, 2010.

${ }^{12}$ Jex, H. R., Magdaleno, R. E., and Junker, A. M., "Roll Tracking Effects of G-vector Tilt and Various Types of Motion Washout," Proceedings of the Fourteenth Annual Conference on Manual Control, 1978, pp. 463-502.

${ }^{13}$ Stapleford, R. L., Peters, R. A., and Alex, F. R., "Experiments and a Model for Pilot Dynamics with Visual and Motion Inputs," Tech. Rep. NASA CR-1325, Systems Technology, Inc., Hawthorne (CA), 1969.

${ }^{14}$ Hosman, R. J. A. W., Pilot's Perception and Control of Aircraft Motions, Ph.D. thesis, Delft University of Technology, Faculty of Aerospace Engineering, 1996.

${ }^{15}$ Van der Vaart, J. C., Modelling of Perception and Action in Compensatory Manual Control Tasks, Ph.D. thesis, Delft University of Technology, Faculty of Aerospace Engineering, 1992.

${ }^{16}$ Mulder, M., Cybernetics of Tunnel-in-the-Sky Displays, Ph.D. thesis, Delft University of Technology, Faculty of Aerospace Engineering, 1999.
} 
${ }^{17}$ Zaal, P. M. T., Pool, D. M., Chu, Q. P., Van Paassen, M. M., Mulder, M., and Mulder, J. A., "Modeling Human Multimodal Perception and Control Using Genetic Maximum Likelihood Estimation,” Journal of Guidance, Control, and Dynamics, Vol. 32, No. 4, 2009 , pp. $1089-1099$.

${ }^{18}$ Gouverneur, B., Mulder, J. A., Van Paassen, M. M., Stroosma, O., and Field, E. J., "Optimisation of the SIMONA Research Simulator's Motion Filter Settings for Handling Qualities Experiments," Proceedings of the AIAA Modeling and Simulation Technologies Conference and Exhibit, Austin, Texas, Aug. 11-14, 2003, No. AIAA-2003-5679, American Institute of Aeronautics and Astronautics, Aug. 2003.

${ }^{19}$ Steurs, M., Mulder, M., and Van Paassen, M. M., "A Cybernetic Approach to Assess Flight Simulator Fidelity," Proceedings of the AIAA Modelling and Simulation Technologies Conference and Exhibit, Providence (RI), No. AIAA-2004-5442, 2004.

${ }^{20}$ Sinacori, J. B., “The Determination of Some Requirements for a Helicopter Research Simulation Facility," Tech. Rep. NASA-CR-152066, Systems Technology Inc., Sept. 1977.

${ }^{21}$ McRuer, D. T. and Jex, H. R., “A Review of Quasi-Linear Pilot Models,” IEEE Transactions on Human Factors in Electronics, Vol. HFE-8, No. 3, Sept. 1967, pp. 231-249.

${ }^{2}$ Shirley, R. S. and Young, L. R., "Motion Cues in Man-Vehicle Control - Effects of Roll-Motion Cues on Human Operator's Behavior in Compensatory Systems with Disturbance Inputs," IEEE Transactions on Man-Machine Systems, Vol. 9, No. 4, Dec. 1968, pp. $121-128$.

${ }^{23}$ McRuer, D. T., Graham, D., Krendel, E. S., and Reisener, W. J., "Human Pilot Dynamics in Compensatory Systems, Theory Models and Experiments with Controlled Element and Forcing Function Variations," Tech. Rep. AFFDL-TR-65-15, Air Force Flight Dynamics Laboratory, 1965.

${ }^{24}$ Levison, W. H., “A Model for the Pilot's Use of Roll-Axis Motion in Steady-State Tracking Tasks,” BBN Report 3808, Bolt Beranek and Newman Inc., Cambridge (MA), May 1978.

${ }^{25}$ Bryson, Jr., A. E. and Ho, Y.-C., Applied Optimal Control: Optimization, Estimation, and Control, Taylor \& Francis Group, New York (NY), 1975 .

${ }^{26}$ Advani, S. K., The Kinematic Design of Flight Simulator Motion-Bases, Ph.D. thesis, Faculty of Aerospace Engineering, Delft University of Technology, 1998.

${ }^{27}$ Field, E. J., Armor, J. B., and Rossitto, K. F., "Effects of Pitch Instantaneous Center of Rotation Location on Flying Qualities," Proceedings of the AIAA Modeling and Simulation Technologies Conference and Exhibit, Monterey (CA), No. AIAA-2002-4799, 2002.

${ }^{28}$ Berkouwer, W. R., Stroosma, O., Van Paassen, M. M., Mulder, M., and Mulder, J. A., "Measuring the Performance of the SIMONA Research Simulator's Motion System," Proceedings of the AIAA Modeling and Simulation Technologies Conference and Exhibit, San Francisco, California, Aug. 15-18, 2005, No. AIAA 2005-6504, Aug. 2005, pp. 1-12.

${ }^{29}$ Stroosma, O., Van Paassen, M. M., Mulder, M., and Postema, F. N., "Measuring Time Delays in Simulator Displays," Proceedings of the AIAA Modeling and Simulation Technologies Conference and Exhibit, Hilton Head (SC), No. AIAA-2007-6562, 2007.

${ }^{30}$ Damveld, H. J., A Cybernetic Approach to Assess the Longitudinal Handling Qualities of Aeroelastic Aircraft, Ph.D. thesis, Delft University of Technology, Faculty of Aerospace Engineering, May 2009.

${ }^{31}$ Hosman, R. J. A. W., Grant, P. R., and Schroeder, J. A., "Pre and Post Pilot Model Analyis Compared to Experimental Simulator Results," AIAA Modeling and Simulation Technologies Conference and Exhibit, San Francisco (CA), No. AIAA-2005-6303, 2005. 